\title{
Large-eddy simulation of the flow past a circular cylinder at sub- to super-critical Reynolds numbers
}

\author{
Seong Mo Yeon, Jianming Yang, Frederick Stern ${ }^{1}$ \\ IIHR-Hydroscience and Engineering \\ University of Iowa \\ Iowa City, IA 52242, USA
}

\begin{abstract}
Large-eddy simulation of turbulent flow past a circular cylinder at sub- to super-critical Reynolds numbers is performed using a high-fidelity orthogonal curvilinear grid solver. Verification studies investigate the effects of grid resolution, aspect ratio and convection scheme. Monotonic convergence is achieved in grid convergence studies. Validation studies use all available experimental benchmark data. Although the grids are relatively large and fine enough for sufficiently resolved turbulence near the cylinder, the grid uncertainties are large indicating the need for even finer grids. Large aspect ratio is required for sub-critical Reynolds number cases, whereas small aspect ratio is sufficient for critical and super-critical Reynolds number cases. All the experimental trends were predicted with reasonable accuracy, in consideration the large facility bias, age of most of the data, and differences between experimental and computational setup in particular free stream turbulence and roughness. The largest errors were for under prediction of turbulence separation.
\end{abstract}

Key words: Large-eddy simulation, circular cylinder, critical Reynolds number, drag crisis, verification and validation

\section{Introduction}

Physics and simulation capability of three-dimensional (3D) unsteady separation remains a significant challenge for many fields, including marine and ocean engineering. Ships and offshore structures suffer separations due to bluff bodies, sharp edges, appendages, wave-induced, and off-design conditions. A fundamental problem for both applications is the turbulent flow past

${ }^{1}$ Corresponding author. E-mail: frederick-stern@uiowa.edu; Tel.: +1 319-335-5215. 
a circular cylinder studied for many years focusing on Reynolds number $(\mathrm{Re})$ effects on smooth surface separation and wake.

The following flow regimes for the turbulent flow past a circular cylinder were defined in Williamson (1996), Sumer (2006), and Schewe (1983): sub-critical for $3 \times 10^{2}<\operatorname{Re}<2 \times 10^{5}$, critical for $2 \times 10^{5}<\operatorname{Re}<3.5 \times 10^{5}$, super-critical for $3.5 \times 10^{5}<\operatorname{Re}<1.5 \times 10^{6}$, and post-critical for $\operatorname{Re}>1.5 \times 10^{6}$. Laminar/turbulent separation, laminar separation bubble, turbulent transition, shear layer and Karman instabilities govern the nature of the flow separation for different flow regimes. In particular, the sudden drop of drag force in the critical Re regime, i.e., drag crisis phenomenon, is one of the important topics in fluid dynamics due to its complex flow physics.

For experimental studies, flow phenomena including instabilities as per flow region were explained and summarized in detail by Williamson (1996). Local pressure and skin-friction coefficients were measured at a wide range of Re from sub-critical to post-critical region and boundary layer separation and transition from laminar to turbulent was characterized by Achenbach (1968). At high Re, super-critical region, drag coefficient and vortex shedding frequency was measured and studied by Roshko (1961). Turbulent flow at critical region was studied by Bearman (1969) and Farell and Blessmann (1983). Pfeil and Orth (1990) studied the influence of the flow disturbances on the separation and transition of the boundary layer and measured flow transition near the laminar separation bubble for the super-critical Re. Experimental benchmark validation data are available for mostly global/integral and limited local flow variables, as summarized in Table 1.

There were many computations for flow around a circular cylinder at a wide range of Re. Most of them were focused on the low Re range and provided good results. However, there were few studies conducted at critical Re including RANS/URANS (unsteady Reynolds Averaged Navier-Stokes Simulation), DES (Detached Eddy Simulation) and LES (Large Eddy Simulation). URANS methods cannot predict the drag crisis due to its inability to predict boundary layer transition to turbulence since most studies either neglect transition modeling or use models that do not display correct trends (Vaz et al. 2007; Moussaed et al. 2013; Wen and Qui; 2013; Ye et al. 2013). For instance, Vaz et al. (2007) investigated drag crisis in two/three dimensions using RANS approach. A good agreement of drag coefficient was obtained at the sub-critical region but the results were unsatisfactory at the super-critical region. Even a clear drop of drag coefficient was 
not found. Although the Stouhal number $(\mathrm{St})$ was predicted well, this cannot guarantee accurate flow solution as pointed out by Rodi et al. (1997). Hybrid methods such as DES show some improvements over URANS, but only few studies to reach definitive conclusions (Vaz et al. 2007; Moussaed et al. 2013; Travin et al. 2000). Simulations with laminar and turbulent separations was performed at sub-critical and super-critical Re with DES by Travin et al. (2000) and a good agreement of local pressure distribution was obtained but there was discrepancy in the skin-friction distribution at super-critical Re which implies the laminar to turbulent transition in the boundary layer from his approach. The few LES (large-eddy simulation) studies on the flow past a circular cylinder show promising results for predicting the drag crisis.

A LES study was performed at a Re in the subcritical region, but very close to critical region, by Breuer (2000). In his study, SGS (subgrid scale stress) model and aspect ratio effects were examined and good agreement was obtained, especially in the near wake although his grid refinement did not show much improvement. Another LES study was conducted at the supercritical region with a wall-modeled boundary layer by Catalano et al. (2003). In their study, the skin-friction had a similar discrepancy as Travin et al. (2000)'s results due to the inadequately modeled boundary layer. Recently, a LES study at the critical region was performed by Ono and Tamura (2008) showed good agreement for the super-critical Re but lacked other regimes; the flow structures included the laminar separation bubble but separation angle was under-predicted. Studies roughly showed trends of St, but pressure and shear stress distributions were limited. James and Lloyd (2013) predicted the drag crises while Lee and Yang (2013) suggested that capability but lacked super-critical results. Kim and Mohan (2005) showed good agreement for the sub-and super-critical Re but lacked critical results. Current LES only sparsely covers Re regimes up to the super-critical Re, use small AR especially at the sub-critical Re, use relatively coarse grids for the boundary layer, and do not make full use of the available experimental benchmark validation data.

Momentum and energy conserving convection schemes such as central difference schemes are optimum for LES as with sufficient grid quality and resolution enable fully resolved turbulence up to the grid cutoff frequency, whereas non-conserving schemes display numerical dissipation well before the grid cutoff frequency (Mittal and Moin, 1997). However, central difference schemes are unstable on greatly stretched grids; thus, LES for single-phase complex geometries uses momentum conservative third order Quick and $5^{\text {th }}$ order WENO upwind schemes, which are able to capture an acceptable range of the energy cascade (Mittal and Moin, 1997). LES for two- 
phase flows has the additional difficulty of stability across the interface for conservative convection schemes; therefore, non-conservative schemes have been used in particular in the precursory research for surface-piercing cylinder flow by Suh et al. (2011) and Koo et al. (2014) for sub-critical and sub- to super-critical Re, respectively. The sub-critical Re results were satisfactory however the critical and super critical were not since the deep flow did not display the correct single-phase trends.

The objective herein is verification, validation and analysis of physics for high fidelity LES of single-phase cylinder flow for sub- critical and super-critical Re in conjunction with the ITTC OEC Workshop on VIV and Wave Run-up held in Nantes, France October 17-18, 2013. The approach uses an orthogonal curvilinear grid flow solver, CFDShip-Iowa V6.2, quantitative verification \& verification, sensitivity studies for AR, grid and convection schemes, assessment of LES quality, validation using all available experimental data.

\section{Computational methods}

In the LES approach, the Navier-Stokes equations are spatially filtered so that the large, energy carrying eddies are resolved and the small-scale, dissipative eddies are modeled by a SGS model. After applying the filtering operation and the SGS model, the Navier-Stokes equations for the incompressible viscous flow with constant density and viscosity can be written as:

$$
\begin{gathered}
\frac{\partial \overline{\mathbf{u}}}{\partial \mathrm{t}}+\nabla \cdot(\overline{\mathbf{u}} \overline{\mathbf{u}})=-\nabla \overline{\mathrm{p}}+\nabla \cdot\left[\left(\nu+v_{\mathrm{t}}\right)\left(\nabla \overline{\mathbf{u}}+(\nabla \overline{\mathbf{u}})^{\mathrm{T}}\right)\right] \\
\nabla \cdot \overline{\mathbf{u}}=0,
\end{gathered}
$$

where $t$ is the time, $\mathbf{u}$ is the velocity vector, $\mathrm{p}$ is the pressure, the bar on a variable ${ }^{-}$denotes the filtering operation, $v$ is the kinematic viscosity, and superscript $\mathrm{T}$ represents the transpose operation. The turbulent eddy viscosity $v_{\mathrm{t}}$ is defined as

$$
v_{\mathrm{t}}=C \Delta^{2}|\overline{\mathbf{S}}|
$$

where $\Delta$ is the filter length (the implicit top-hat filter in this study), $|\overline{\mathbf{S}}|=\sqrt{2 \overline{\mathbf{S}} \cdot \overline{\mathbf{S}}}$ with the filtered

strain rate tensor $\overline{\mathbf{S}}=\frac{1}{2}\left[\nabla \overline{\mathbf{u}}+(\nabla \overline{\mathbf{u}})^{\mathrm{T}}\right]$, and the coefficient $C$ is to be determined by the SGS model to close the equations. It should be noted that the isotropic component of the SGS stress tensor $(\overline{\mathbf{u}} \mathbf{u}-\overline{\mathbf{u}} \overline{\mathbf{u}})$ is incorporated into the filtered pressure $\overline{\mathbf{p}}$ in Eq. (1). The Lagrangian dynamic Subgrid- 
scale (SGS) model (Meneveau et al., 1996) is adopted in the present study. In this model, the ensemble average is applied along the fluid particle trajectories to determine the model coefficient $C$, which is particularly suitable for complex geometries without the requirement of homogeneous directions. A comparison of the performance of difference SGS models can be found in Sarghini et al. (1999).

The finite-difference method is used to discretize the Navier-Stokes equations on a nonuniform staggered orthogonal curvilinear grid (Yang and Stern, 2009; Suh et al., 2011; Wang et al., 2012). A staggered variable arrangement is adopted where the contravariant velocity components are defined at cell faces in three grid directions, respectively, and all other variables are defined at cell centers. A four-step fractional-step method is employed for velocity-pressure coupling, in which a pressure Poisson equation is solved to enforce the continuity equation. A semi-implicit time advancement scheme is used to integrate the momentum equations with the second-order Crank-Nicolson scheme for the diagonal viscous terms and the second-order Adams-Bashforth scheme for the convective terms and other viscous terms. For the spatial discretization, QUICK (Leonard 1979) and WENO (Jiang and Shu 1996) are used for the approximation of the convective terms. In this study a WENO scheme in non-conservative form (NC-WENO) is also tested, in which the convective terms are written as $\mathbf{u} \cdot \nabla \mathbf{u}$ as used in two-phase flow simulations (Suh et al., 2011). This was added as a means of analyzing the effect of convection schemes in LES of twophase flows. A central difference scheme is used for other terms. Taking advantage of the periodic boundary condition and uniform grid distribution in two spatial directions, a fast Poisson solver as used in Yang and Balaras (2006) is employed for reducing the computational cost substantially. Because of the geometric periodicity and uniform grid spacing in the azimuthal and spanwise directions, in the fast Poisson solver FFTs (fast Fourier transforms) are applied in these two directions; whereas a TDMA (tri-diagonal matrix algorithm) solver is applied in the radial direction. Further details of the computational methods can be found in Yeon (2014).

The parallelization is done via a domain decomposition technique using the MPI library. A simple domain decomposition technique is used where the orthogonal grid is divided into equal blocks, each of which resides in one CPU core. A parallel tri-diagonal system solver is used with the approximate factorization of momentum equations and no iterations are needed for the inversion of the momentum equations. The parallelization of the fast Poisson solver is achieved 
through memory transpositions along different grid directions. Parallel I/O based on MPI 2 is implemented. Instead of the usual approaches that one process collects all data from all processes and write to one file, or, each process write its data to its own file, in the current approach all processes write its data to one single file, which is very scalable and can greatly simplify the I/O operation and minimize the post-processing overhead.

\section{Simulation setup and conditions}

The origin of the coordinates is located at the center of the cylinder with diameter D and span length L. The streamwise, transverse and spanwise directions are set to X, Y, Z, respectively. The flows are computed in a cylindrical coordinate where radial direction $r$ extends from the center of the cylinder, azimuthal direction $\theta$ is in anti-clockwise direction starting from the downstream direction and the axis z coincides with the spanwise direction. The radial outer boundary is divided into inflow and outflow boundaries at $\theta=90^{\circ}$ and $\theta=270^{\circ}$. A Dirichlet boundary condition is applied for the inflow boundary with uniform free stream velocity $U$ and a convective outflow boundary condition is imposed for the outflow boundary. No-slip boundary condition is employed on the cylinder wall and periodic boundary condition is imposed on the top and bottom sides of the cylinder. The distance from the center of the cylinder to the outer boundary is $20 \mathrm{D}$. L varies from $2 \mathrm{D}$ to $8 \mathrm{D}$. The points of the body-fitted grid are uniformly distributed in the spanwise and azimuthal directions. The points in the radial direction are clustered near the cylinder wall to resolve the boundary layer and flow separation.

Table 2 summarizes the simulations setup and conditions including case number, $\mathrm{Re}=\mathrm{UD} / v$ ( $v$ is the kinematic viscosity), grid size, AR, convection scheme and near wall distance $y+$. The cylindrical grids enable very good resolution of the boundary layers and detached shear layers close to the wall. But they become coarser in the azimuthal direction as the radial distance to the cylinder surface increases and a grid refinement in critical wake regions such as the vortex formation region is difficult to achieve without the help of some advanced gridding techniques such patched refinement blocks or adaptive grid refinement. Therefore, 512 points were used in the azimuthal direction in this work to obtain reasonable resolution in these regions even at a radial distance of 2D away from the cylinder surface. Of course, this may result in some waste of computation time due to the excessive azimuthal resolution very close to the wall, but the simpler grid topology enables a faster pressure solver and thus an overall better cost-effectiveness. Initially, 
simulations were performed for $\mathrm{AR}=2$ with medium grid, based on $\mathrm{AR}$ values used in previous simulations. The medium grid has about 67 million grid points. Subsequently additional simulations were performed to study the effects of AR, grid resolution (134 and 8 million grid points for fine and coarse grid, respectively) and convection scheme. For sub-critical Re, cases 17 are for $\operatorname{Re}=6.31 \times 10^{4}$ and cases $8-13$ are for $\operatorname{Re}=1.26 \times 10^{5}$. For critical Re, cases $14-19$ are for $\operatorname{Re}=2.52 \times 10^{5}$. For super-critical $\operatorname{Re}$, case 20 is for $\operatorname{Re}=4.15 \times 10^{5}$; cases $21-22$ are for $\operatorname{Re}=5.06 \times 10^{5}$ and cases $23-30$ are for $\operatorname{Re}=7.57 \times 10^{5}$. Cases $5,12,18,20,21$, and 26 were submitted to the ITTC workshop. Cases 23-25 are for quantitative verification and validation. The $\mathrm{y}^{+}$corresponding to the first grid point is 0.67 for the coarse grid and 0.15 for the fine grid at $\operatorname{Re}=7.57 \times 10^{5}$. The $y^{+}$ value decreases as Re decreases and reach to 0.03 at $\operatorname{Re}=6.31 \times 10^{4}$. For all cases, $512 \mathrm{CPU}$ cores are used for the computation. Each case takes over 2500 wall clock hours; in which about 1500 hours for reaching statistically stationary solutions and about 1000 hours for covering 20 Karman vortex-shedding cycles. A constant time step $\Delta \mathrm{t}=1 \times 10^{-4}$ is used.

Yeon (2014) provides details of the analysis methods, which include quantitiative verification and validation; cross correlation coefficient and correlation length; energy spectra in the shear layer and wake, grid cuttoff and Kolmorogorov scales; and shear layer instability.

\section{Results}

\subsection{Quantitative verification and validation and sensitivity studies}

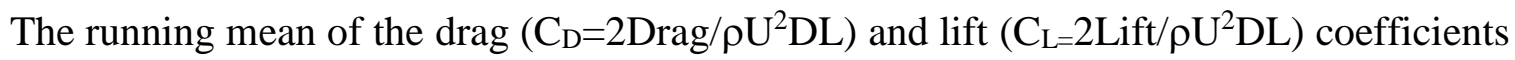
time histories are used to determine statistical convergence. When the fluctuations of the running mean are smaller than $1 \%$ of the mean value, the flow is statistically stationary. The analysis uses about 20 vortex-shedding cycles after the flow reaches the stationary condition, as specified by the OEC.

Quantitative verification and validation for $\mathrm{Re}=7.57 \times 10^{5}$ follows Stern et al. (2006) and Xing and Stern (2010). The QUICK is computationally less expensive than the WENO scheme and used. In the following, T, S, and E represent the experimental data, simulation results, and comparison error, respectively. The facility bias uncertainty is defined as the half of the difference of the maximum and minimum of the experimental data, and $\mathrm{U}_{\mathrm{FB}}=5 \% \mathrm{~T}$. The grids denoted by $\mathrm{F}$, $\mathrm{M}$ and $\mathrm{C}$ represent fine, medium and coarse grids systematically refined by a refinement ratio of 2 
and the simulation results are marked as $S_{1}, S_{2}$, and $S_{3}$, respectively. The grid uncertainty for $C_{D}$, $\mathrm{C}_{\mathrm{L}}{ }^{\mathrm{RMS}}$ and $-\mathrm{C}_{\mathrm{pb}}$ are $\mathrm{U}_{\mathrm{G}}=24.9 \% \mathrm{~S}_{1}, 50.4 \% \mathrm{~S}_{1}$ and $0.34 \% \mathrm{~S}_{1}$, respectively, which are relatively large and indicate that much finer than the current fine grid (67M) are required for the super-critical Re. The comparison error for the $\mathrm{C}_{\mathrm{D}}$ is $\mathrm{E}=2.8 \% \mathrm{~T}$. The comparison error for the $\mathrm{C}_{\mathrm{L}}{ }^{\mathrm{RMS}}$ is $\mathrm{E}=4.0 \% \mathrm{~T}$. The comparison error for the $-\mathrm{C}_{\mathrm{pb}}$ is $\mathrm{E}=1.4 \% \mathrm{~S}_{1}, \mathrm{C}_{\mathrm{D}}, \mathrm{C}_{\mathrm{L}}{ }^{\mathrm{RMS}}$ and $-\mathrm{C}_{\mathrm{pb}}$ display monotonic convergence with estimated orders of accuracy of $\mathrm{p}_{\mathrm{RE}}=1.4,1.6$ and 1.8, respectively, which approach the theoretical second order accuracy of the numerical method in this work.

Sensitivity study was conducted for the effect of AR, grid resolution and convection scheme for the $\mathrm{C}_{\mathrm{D}}, \mathrm{C}_{\mathrm{L}}{ }^{\mathrm{RMS}}$ and $-\mathrm{C}_{\mathrm{pb}}$. $\mathrm{AR}=8$ gave the best results for the sub-critical Re. $\mathrm{AR}=8$ gave better results in terms of $C_{D}$ for the critical Re. $A R=2$ gave the best results for the super-critical Re. For the convection scheme, the results for the sub-critical Re are not conclusive since the AR was small. For the critical Re, the QUICK scheme showed early drag crisis compared with experimental data and WENO with the same AR. For the super-critical Re, conservative WENO showed better results. Cases 5 and 12 for the sub-critical, case 18 for the critical and cases 20, 21 and 26 for the super critical Re were submitted to the OEC (Yeon et al., 2013).

Three-dimensional effect of solutions is examined with the cross correlation coefficient of pressure (Szepessy, 1994) as the following form:

$$
\mathrm{R}_{\mathrm{pp}}=\frac{\overline{\mathrm{p}_{\mathrm{i}} \mathrm{p}_{\mathrm{j}}}}{\sqrt{\overline{\overline{\mathrm{p}_{\mathrm{i}}^{2}}} \sqrt{\overline{\mathrm{p}_{\mathrm{j}}^{2}}}}}
$$

where subscript $\mathrm{i}$ is located at midspan and subscript $\mathrm{j}$ is distanced from $\mathrm{i}$ along the span. The estimated correlation coefficients are integrated over spanwise direction to get correlation length (Norberg, 2001),

$$
\Lambda=\int \mathrm{R}_{\mathrm{pp}}(z) \mathrm{dz}
$$

where $\mathrm{z}$ is the location in the spanwise direction. The computed correlation length is examined to determine proper span length for the present study. Figure 1(a) and (b) show the correlation coefficient for $\operatorname{Re}=4.3 \times 10^{4}$ and $2.4 \times 10^{5}$ and correlation length against $\mathrm{Re}$. The correlation coefficient rapidly decreases as $\Delta \mathrm{z}$ increases. The correlation length divided by $\mathrm{D}$ drops from 10 for $\mathrm{Re}=10^{3}$ to 3 for the end of the sub-critical Re, suddenly precipitate to 1.5 for the critical Re and stays at that value for the super- and post-critical Re. Thus, large AR domains are required for 
CFD simulations for the sub-critical Re, e.g. $\mathrm{L} / \mathrm{D}$ is about 5 for $\mathrm{Re}=10^{4}$. The comparison for the correlation coefficient with experiments for sub- and critical Re along with LES results for sub-, critical and super-critical Re is also given. The LES are all for AR =8, i.e., cases 5, 12, 18, and 29. For sub- and critical Re, the LES shows similar trends (increasing slope) as the data but with scatter. For critical and super-critical Re, the LES suggests that the slope continues to increase with Re, but here again the LES results show scatter. Fig. 1(b) shows the comparison for the correlation length with experiments for sub- and critical Re along with LES results for sub-, critical and super-critical Re. For sub- and super critical Re, the LES is close to the data and empirical formula, whereas it under-predicts the data/formula for critical Re.

\subsection{Integral and local variables}

Figure 2(a), (b), (c), (d), (e), and (f) show the drag coefficient $C_{D}$, lift (mean side force) coefficient $C_{L}$, RMS lift coefficient $C_{L}{ }^{\text {RMS }}$, separation angle $\theta_{s}$, base pressure coefficient $-C_{p b}$, and Strouhal number St against Re, respectively. The drag coefficient shows clear trends albeit with fairly large $10 \%$ average value scatter between the experimental facilities due to many factors including aspect ratio (AR), free stream turbulence, roughness, blockage, etc. The drag coefficient is nearly constant for the sub-critical $\operatorname{Re} C_{D} \approx 1.2$, drops dramatically (drag crisis) by a factor of 4 to $\mathrm{C}_{\mathrm{D}} \approx 0.28$ for the critical $\mathrm{Re}$, nearly constant at this value for the super-critical Re, and increases by a factor of 2.5 up to $\mathrm{C}_{\mathrm{D}} \approx 0.7$ for the post-critical Re. The drag crisis is well predicted in the present LES, although more cases in the critical and post-critical regime are desirable. In experiments, the mean lift $\mathrm{C}_{\mathrm{L}}$ is mostly zero except for border of critical- and super-critical $\mathrm{Re}$ where some experiments show large $\mathrm{C}_{\mathrm{L}} \approx 1.25$ due to asymmetric laminar separation bubble condition. The LES $C_{L}$ shows nearly zero values similarly as the experiments, except those with asymmetric laminar separation bubbles near the boundary of critical Re. On the other hand, the RMS lift coefficient is large $\left(\mathrm{C}_{\mathrm{L}}{ }^{\mathrm{RMS}} \approx 0.4\right)$ for the sub-critical Re, drops dramatically by a factor of 10 to $\mathrm{C}_{\mathrm{L}}{ }^{\mathrm{RMS}} \approx 0.04$ for the critical $\mathrm{Re}$, nearly constant at this value for the super-critical Re, and increases by factor of 2 to $\mathrm{C}_{\mathrm{L}}{ }^{\mathrm{RMS}} \approx 0.08$ for the post-critical Re. The differences between RMS lift coefficient between facilities for the sub-critical Re is due to differences between use of load cell (lower values) and pressure-integration (higher values) measurement systems. The LES $C_{L}{ }^{R M S}$ is close to the most reliable data for sub-, critical and super-critical Re. The separation angle moves from $\theta_{\mathrm{s}} \approx 75$ to $90 \mathrm{deg}$ for the sub-critical Re, increases dramatically up to $\theta_{\mathrm{s}} \approx 140$ 
deg for the critical Re, nearly constant at this value for the super-critical Re, and decreases to $\theta_{\mathrm{s}} \approx$ $125 \mathrm{deg}$ for the super-critical Re. In the present LES, the angle of separation is close to the experiments for sub- and critical Re, but substantially under predicted for super-critical Re. The base pressure trends in experiments closely follow the drag trends. The base suction pressure in LES shows good agreement with the experiments for sub- and super-critical Re, but is under predicted for critical Re. In the experiments, the St is nearly constant $S t \approx 0.2$ for the sub-critical $\mathrm{Re}$, increases dramatically to St $\approx 0.475$ for the critical Re, drops to St $\approx 0.425$ for the supercritical $\mathrm{Re}$, and dramatically drops to $\mathrm{St} \approx 0.1$ for initial post-critical Re and increases up to St $\approx 0.3$ for Re up to nearly $10^{7}$. The LES St is close to the experiments for sub- and critical Re, but under-predicts for super-critical Re. Since time history of lift coefficient is highly correlated to St, the under-estimation of St for the super-critical Re is likely due to grid coarseness. Tables 3 and 4 summarize the experimental data $(\mathrm{T})$, simulation results $(\mathrm{S})$, comparison error (E) and facility bias $\left(\mathrm{U}_{\mathrm{FB}}\right)$ values for all six selected cases. For $\mathrm{C}_{\mathrm{D}}$ the average comparison error is $\mathrm{E}=$ $22 \% \mathrm{~T}$, which is over twice as large as $\mathrm{U}_{\mathrm{FB}}=10 \% \mathrm{~T}$. The largest comparison error is found in critical Re, where the drag drops sharply with small changes in Re resulting in large changes in $C_{D}$ between facilities and likely simulations. The comparison error for the other variables are also relatively large that is average $\mathrm{E}=6 / 38,27,7$ and $7 \% \mathrm{~T}$ for $\theta_{\mathrm{LS}} / \theta_{\mathrm{TS}},-\mathrm{C}_{\mathrm{pb}}, \mathrm{C}_{\mathrm{L}}^{\mathrm{RMS}}$ and $\mathrm{St}$, respectively.

Figure 3 compares the LES surface pressure, shear stress and angular pressure gradient distributions against the experimental data for the three selected cases along with experimental data for a post-critical Re. As summarized in Table 5, key point values are defined for minimum pressure (MP), laminar separation (LS), turbulent transition (TT), turbulent reattachment (TR), turbulent separation (TS) and base pressure (BP) positions. LS is where the shear stress changes from positive to negative. A large rate of turbulence intensity $>20 \%$, which occurs outside the separation bubble boundary at its maximum thickness, defines TT. The location that the shear stress recovers to a local maximum after separation defines TR, which generally corresponds to the end of surface pressure plateau. Where the shear stress changes from positive to negative or where it becomes constant after LS defines TS. BP is at $180 \mathrm{deg}$ from stagnation point. Overlaid on the shear stress distributions is the angular pressure gradient $\mathrm{dp} / \mathrm{d} \theta$ determined from numerical differentiation of the pressure distributions. The differences between facilities are due to Re values and regimes transition differences. These differences are relatively small for the sub-critical 
compared with the critical, super- and post-critical Re. The LES shows fairly close agreement with the experiments in spite of the Re differences, including details such as the laminar separation bubble. The key points and their comparison errors are summarized in Tables 6 and 7, respectively. The trends qualitatively agree with the experiments. The LES comparison error for LS is $\mathrm{E}=6 \% \mathrm{~T}$, but MP, TR, TS are mostly under-predicted with average comparison error E = 17, 15 and 38\% T, respectively. The large comparison error for TS could be attributed to the turbulence intensity of the freestream and the roughness of the cylinder in the experiments; however, LES numerical methods and grid refinement are also issues.

\subsection{Mean flow field}

As shown in Fig. 4, the mean streamlines show closed-separation for all Re with wide long bubble for sub, narrow shorter bubble for critical and narrow long bubble for super critical Re. Close-up view of the mean flow field near the separation area for each Re is also shown. For regime change from sub- to critical Re, LS moves downstream with increasing distance from MP. For super-critical Re, MP/LS coincide and move further downstream followed by the formation of a laminar separation bubble, TT, TR and TS. Following Pfeil and Orth (1990), the TT is determined by the turbulence intensity level and rate across the maximum boundary of the bubble. For subcritical Re, the laminar flow is observed before LS and maintained for the beginning part of the separated flow. Turbulent transition occurs in the shear layer and the maximum value appears after the separation at around $x=0.3$. The boundary layer and some part of separated boundary layer are laminar. For critical Re, the laminar flow is observed right after LS followed by turbulent transition. A secondary vorticity is formed after the LS but reattachment is not observed in the mean flow field. The turbulent transition occurs through the secondary vorticity and the turbulence intensity increases as flow goes downstream. For super-critical Re, the laminar flow is observed up to TT. A separation bubble is formed right after LS and TR is observed at the end of the bubble. The turbulent transition appears across the separation bubble followed by TR as shown by Pfeil and Orth (1990). For the sub-critical Re, a very small laminar separation bubble with a laminar reattachment was observed. For the critical and super-critical Re, the separated flow beginning at LS forms a secondary vorticity close to the wall, which amalgamates into recirculation bubble without reattaching to the wall in the mean flow field. The mean shear layer as identified by the turbulent intensity contours moves to the cylinder wall as Re increases and the transition point of 
the shear layer, the point where first shedding was found, moves upstream up to the separation point. For the super-critical Re, the boundary between shear layer and boundary layer is hard to distinguish and the layers are close enough to interact.

Fig. 5(a) shows the laminar separation bubble, which is defined by the zero contour line of the mean axial velocity and reduces in size or even disappears for increased levels of free stream turbulence. For post critical Re, MP and TS move upstream. The boundary layer transition from laminar to turbulent flow delays separation, which narrows the wake and reduces the drag. The narrower the wakes the smaller the RMS lift, but the larger the St. Fig. 5(b) shows the LES mean velocity and turbulence intensity near the separation bubble and key point and maximum thickness values are shown in Table 8. Since the diameter is non-dimensionalized in the LES, the figure is dimensionalized with the diameter $300 \mathrm{~mm}$ as in the experiment (Pfeil and Orth, 1990). The separation bubble is distinguishable by the zero mean velocity level, which is tear drop shaped initiating at LS (94 deg), maximum at TT (102 deg), and terminating at TR (105 deg); the comparison errors for LS, TT and TR are E=-6, -7 and $-8 \% \mathrm{~T}$, respectively. The transition of the separated shear layer is indicated by the sudden increase in the turbulence intensity at around 102 deg. The LES mean velocity and turbulence intensity contours shows similar distribution to that of the experiment with early formation of the separation bubble. It should be noted that the flow condition including $\mathrm{Re}$, roughness and freestream turbulence is not identical between the experimental and the present simulation. The discrepancy between the present LES study and experiment is attributed in part to the differences. The separation bubble reaches its maximum thickness at around $102 \mathrm{deg}$ and the thickness is about 0.05/300. Even though the location of separation bubble is formed earlier in the present LES, the thickness and the level of turbulent intensity are almost identical to the experiment. The angle from LS to TT and to TR are 8 and 11, respectively and almost identical to experimental data; the comparison errors are $\mathrm{E}=-20$ and $-21 \% \mathrm{~T}$, respectively, where small changes can result in relatively large comparison errors due to small angle.

Figure 6 shows the comparison of the resolved Reynolds stresses with the experiments for sub-critical Re along with LES results for sub-, critical and super-critical Re. In particular, Reynolds stresses u'v' across the wake at $x=1 D$ downstream station and u'u' and v'v' along the wake centerline for $0<X / D<8$ are given. The u'v' is anti-symmetric about $y=0$ with maximum values \pm 0.12 . The $u^{\prime} u$ ' is maximum near $1.2 \mathrm{D}$ and rapidly decreases until $2 \mathrm{D}$ followed by gradual 
decrease. The v'v' shows similar trends as u'u' with its maximum at 1.6D. The average comparison errors for u'v', u'u' and v'v' are E=9, 12 and 30\% T for sub-critical Re, which is partially due to Re differences. In general the LES over-predicts the experimental data. From the figure it is evident that the Reynolds stress magnitudes decrease with Re. The resolved Reynolds stresses presented here may include the effects of vortex shedding (Parnaudeau et al., 2008), which is stronger and more coherent in the sub-critical regime, due to the limited sample size (20 vortex shedding cycles). It should be noted that the modeled part of the Reynolds stress tensor by the SGS model is not included in the figure. In Cantwell and Coles (1983) the Reynolds stresses were separated into a periodic mean component and a random component. Although not pursued in this work, such a decomposition of the Reynolds stresses can be useful in the analysis of the results.

\subsection{Instantaneous flow field}

Figure 7 shows the instantaneous vorticity for the sub-, critical and super-critical Re. The shedding vortices are apparent and large structures are observed downstream for the sub-critical Re. The shedding is observed from $x=1 D$ due to the short recirculation region. The shedding vortices are observed farther downstream from $\mathrm{x}=1.5 \mathrm{D}$ for the critical and super-critical Re due to the elongated wake region. As evident in the close-up view, the mean center of shear layer becomes close to the separation bubble as Re increases. The movement of the shear layer to the wall increases chance to interact with boundary layer. As a result, the boundary layer is mixed with the shear layer for the super-critical Re. This mixing of two layers results in the reattachment of the separated flow and forms the separation bubble as Re increases. It should be noted the vortex formation region is highly sensitive to the grid resolution. In the current simulations, 512 grid points were used in the azimuthal direction. In general, the grid resolution in this region is satisfactory for the sub- and critical Re cases, but might be inadequate after 2D away from the cylinder for the super-critical Re cases.

As shown in Fig. 8, vortex shedding in the sub-critical region is apparent and large structures are visible in downstream. But in the super-critical region, relatively small vortex structures were observed. Vortex structures are more clearly shown with iso-surface of Q criterion (Hunt et al., 1988) colored by eddy viscosity. Eddy viscosity, $v_{t}$ was non-dimensionalized with molecular viscosity, $v$, and $v_{t}^{+}=v_{t} / v$. Large scale motion, alternative vortex shedding at subcritical Re is clearly visible in the near wake while chaotic flow appears at near wake. On the 
contrary to the sub-critical case, wake region gets narrow and chaotic even downstream at supercritical Re so that large scale vortex shedding is not observed. It should be noted that, in this work the coarse and medium grids were designed to resolve the boundary layers without considering the resolution requirement in the wake. For this reason we didn't look into how the size of the structures varies with grid resolution by comparing results from different grids. So it is unclear whether these large structures converged according to the size of the spatial filter in the LES. This topic will be addressed in future work.

\section{Conclusions and future research}

Large-eddy simulation of the flow past a circular cylinder from sub-critical to super-critical Re regions has been performed using a Lagrangian dynamic SGS model. The present study showed that drag coefficients change dramatically as Re increases through critical region. The quantitative verification and validation study has been carried out. In verification study for the case $\operatorname{Re}=7.57 \mathrm{e}$, large grid uncertainty for the drag and RMS of lift was found, which indicates finer grid is needed. A sensitivity study was conducted for aspect ratio, grid resolution and convection scheme. Definitely, large aspect ratio $(\mathrm{AR}=8)$ produced best results for the sub-critical Re. Small dependency on both aspect ratio and grid resolution was observed for the critical Re. Small aspect ratio $(\mathrm{AR}=2)$ and conservative scheme produced best results for the super-critical Re. However, the verification and validation study was not sufficient and systematic. Thus, more comprehensive and systematic studies are needed, especially for the sub-critical Re using large aspect ratio.

Validation study has been conducted in order to prove the validity of the numerical models. For the sub-critical Re, the LES solutions showed fairly good agreement. For the critical Re, largest error was observed due to the sharp behavior of the variable including drag. For the super-critical Re, some discrepancy including early prediction of turbulent separation was observed due to the grid uncertainty and numerical setup different from experimental setup. Since the validation data are outdated and sparse, new comprehensive experimental studies are needed, especially for local flow measurements.

Further analysis has been conducted with the validated flow solutions. Basic flow features including mean velocity and vorticity showed the change of wake length due to the delayed separation with increasing Re. The main cause of the drag change was attributed to the mean pressure differences between the stagnation and base point. Instantaneous flow features including 
velocity, vorticity and three-dimensional flow structures showed large amplitude and anisotropic flow structures for the sub-critical Re and small amplitude and chaotic flow structures for the critical and super-critical Re. The wake region became narrow and chaotic even downstream for the super-critical Re so that large scale vortex shedding was not observed. The formation of secondary vortex and development into the separation bubble were observed with streamlines near the body and secondary vorticity contour.

To further clarify the influences of different parameters on the results, an extensive study is required with the same grid resolution in the spanwise direction for different spanwise lengths. Other aspects including time step and LES modeling have to be considered too. The study also provides a solid basis for future study on two-phase turbulent flow past a surface-piercing circular cylinder at supercritical Re. Many interesting results were presented in our previous work (Koo et al. 2014) although with an under-resolved boundary layer. As a complementary research of the two-phase flows past a circular cylinder including cases with a swaying motion of the cylinder, experiments in a towing tank for detailed local flow measurements using tomographic PIV as well as force and moment and wave elevation are under way.

\section{Acknowledgement}

This work was sponsored by the Office of Naval Research (ONR) under Grant No. N000141-01-00-1-7, with Drs. Ki-Han Kim and Thomas C. Fu as the program manager. The calculations presented in this paper were performed at the Department of Defense (DoD) Supercomputing Resource Centers (Navy DSRC) through the High Performance Computing Modernization Program (HPCMP). The authors are grateful to the anonymous reviewers for their valuable comments and constructive suggestions.

\section{References}

1. Achenbach, E. (1968). Distribution of local pressure and skin friction around a circular cylinder in cross-flow up to $\mathrm{Re}=5 \times 106$. Journal of Fluid Mechanics, 34: 625-639.

2. Bearman, P. W. (1969). On vortex shedding from a circular cylinder in the critical Reynolds number regime. Journal of Fluid Mechanics, 37: 577-585.

3. Bearman, P.W., Wadcock, A.J. (1973). The interaction between a pair of circular cylinders normal to a stream. Journal of Fluid Mechanics. 61: 499-511. 
4. Bloor, M. S. (1964). The transition to turbulence in the wake of a circular cylinder. J. Fluid Mech. 19: 290.

5. Breuer, M., "A Challenging Test Case for Large Eddy Simulation: High Reynolds Number Circular Cylinder Flow," International Journal of Heat and Fluid Flow, Vol. 21, No. 5, 2000, pp. 648-654.

6. Bruun, H.H., Davies, P.O.A.L. (1975). An experimental investigation of the unsteady pressure forces on a circular cylinder in a turbulent cross flow. Journal of Sound and Vibration. 40: 535-559.

7. Cantwell, B. and D. Coles (1983). An experimental study of entrainment and transport in the turbulent near wake of a circular cylinder. Journal of Fluid Mechanics, Cambridge Univ Press. 136: 321-374.

8. Catalano, P., M. Wang, G. Iaccarino, and P. Moin., "Numerical Simulation of the Flow Around a Circular Cylinder at High Reynolds Numbers," International Journal of Heat and Fluid Flow, Vol. 24, No. 4, 2003, pp. 463-469.

9. Fage, A. and V. M. Falkner (1931). Further Experiments on the Flow Around a Circular Cylinder. Aeronautical Research Council Reports and Memoranda 1, No. 1369, 186-195.

10. Farell, C, and J Blessmann., "On Critical Flow Around Smooth Circular Cylinders," Journal of Fluid Mechanics, Vol. 136, No. 1, 1983, pp. 375-391.

11. Hunt, J., A. A. Wray and P. Moin (1988). Eddies, streams, and convergence zones in turbulent flows. Studying Turbulence Using Numerical Simulation Databases, 2. 1: 193208

12. Iida, A., Otaguro, T., Kato, C., Fujita, H. (1997). Prediction of aerodynamic sound spectra from a circular cylinder. In: S. of Instrument, C. E. of Japan (Eds.), Proceedings of the 5th Triennial International Symposium on Fluid Control, Measurement and Visualization (FLUCOME '97), Vol. 1. Hayama, Japan, 1-4 September, 1997, 126-131.

13. James, M. and Lloyd, T. (2013). Large Eddy Simulations of Circular Cylinders at a range of Reynolds Numbers. Proceedings of the 27th ITTC Workshop on Wave Run-up and Vortex Shedding.

14. Jiang, G.-S. Shu, C.-W., "Efficient implementation of weighted ENO schemes," Journal of Computational Physics, Vol. 126, 1996, pp. 202-228.

15. Kacker, S.C., Pennington, B., Hill, R.S. (1974). Fluctuating lift coefficient for a circular cylinder in cross flow. Journal Mechanical Engineering Science 16: 215-224

16. Kim, S.-E., L. S. Mohan (2005). Prediction of unsteady loading on a circular cylinder in high Reynolds number flows using large eddy simulation. ASME 2005 24th International Conference on Offshore Mechanics and Arctic Engineering: 775-783. 
17. Koo, B. Yang, J., Yeon, S, Stern, F. (2014), Reynolds and froude number effect on the flow past an interface-piercing circular cylinder, International Journal of Naval Architecture and Ocean Engineering. Volume 6, Issue 3, Pages 529-561.

18. Kourta, A., Boisson, H. C., Chassaing, P., Ha Minh, H. (1987). Nonlinear interaction and the transition to turbulence in the wake of a circular cylinder. J. Fluid Mech. 181: 141.

19. Lee, K. and Yang, K.S. (2013). Large Eddy Simulation of Turbulent Fow Past a Circular Cylinder. Proceedings of the 27th ITTC Workshop on Wave Run-up and Vortex Shedding.

20. Leehey, P., Hanson, C.E. (1971). Aeolian tones associated with resonated vibration. Journal of Sound and Vibration 13: 465-483

21. Leonard, BP., "A Stable and Accurate Convective Modelling Procedure Based on Quadratic Upstream Interpolation," Computer Methods in Applied Mechanics and Engineering, Vol. 19, No. 1, 1979, pp. 59-98.

22. Maekawa, T. \& Mizuno, S. (1967). Flow around the separation point and in the near-wake of a circular cylinder. Phys. Fluids Suppl. S184.

23. Meneveau, C., Lund, T. S. and Cabot W.H. (1996), A Lagrangian dynamic subgrid-scale model of turbulence. Journal of Fluid Mechanics, 319:353-385

24. Mittal, R., Moin, P. (1997), Suitability of Upwind-Biased Finite Difference Schemes for Large-Eddy Simulation of Turbulent Flows, AIAA, 35:1415-1417

25. Moeller, M.J. (1982). Measurement of unsteady forces on a circular cylinder in cross flowat subcritical Reynolds numbers, Ph.D. Thesis, Massachusetts Institute of Technology, USA

26. Moussaed, C., M. V. Salvetti, S. Wornom, B. Koobus and A. Dervieux (2013). Simulation of the flow past a circular cylinder in the supercritical regime by blending RANS and variational-multiscale LES models. Journal of Fluids and Structures.

27. Norberg, C. (1987). Effects of Reynolds number and a low-intensity freestream turbulence on the flow around a circular cylinder. Dept of Appl Thermodynamics and Fluid Mech.

28. Norberg, C. (2001). Flow around a circular cylinder: aspects of fluctuating lift. Journal of Fluids and Structures, Elsevier. 15: 459-469

29. Novak, M., Tanaka, H. (1977). Pressure correlations on a vibrating cylinder. In: Eaton, K.J. (Ed.), Proceedings of the 4th International Conference on Wind Effects on Buildings and

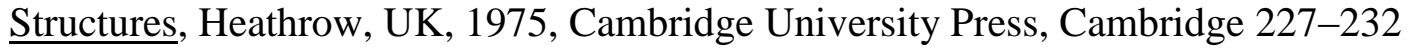

30. Okamoto, S., Hirose, T. \& Adachi, T. (1981). The effect of sound on the vortex-shedding from a circular cylinder. Bull. JSME 24: 45. 
31. Ono, Y. and T. Tamura (2008). LES of flows around a circular cylinder in the critical reynolds number region. Proc. 6 th International Conference on Bluff Body Aerodynamics and Applications: 1-10.

32. Parnaudeau, P., Carlier, J., Heitz, D., Lamballais, E., "Experimental and numerical studies of the flow over a circular cylinder at Reynolds number 3900." Physics of Fluids, 20, 085101 (2008), DOI:http://dx.doi.org/10.1063/1.2957018.

33. Peng, W., Wei, Q. (2013). Numerical Studies of VIV of a Smooth Cylinder. Proceedings of the 27th ITTC Workshop on Wave Run-up and Vortex Shedding.

34. Pfeil H. and Orth U. (1990), Boundary-layer transition on a cylinder with and without separation bubbles. Experiments in Fluids, 10:23-32

35. Rodi, W, JH Ferziger, M Breuer, M Pourquie., "Status of Large Eddy Simulation: Results of a Workshop," Transactions-American Society of Mechanical Engineers Journal of Fluids Engineering, Vol. 119, 1997, pp. 248-262

36. Roshko, A. (1953). On the drag and shedding frequency of two-dimensional bluff bodies. National Advisory Committee for Aeronautics, NACA TN 3169. (Th. Exp. Notch hodograph theory). 3,5.

37. Roshko, A. (1961). Experiments on the flow past a circular cylinder at very high Reynolds number. Journal of Fluid Mechanics, Cambridge Univ Press. 10: 345-356.

38. Sarghini, F., U. Piomelli, E. Balaras (1999). Scale-similar models for large-eddy simulations. Physics of Fluids. 11: 1596-1607.

39. Schewe, G. (1983). On the force fluctuations acting on a circular cylinder in crossflow from subcritical up to transcritical Reynolds numbers. Journal of Fluid Mechanics, Cambridge Univ Press. 133: 265-285.

40. Shih, W., C. Wang, D. Coles and A. Roshko (1993). Experiments on flow past rough circular cylinders at large Reynolds numbers. Journal of Wind Engineering and Industrial Aerodynamics, Elsevier. 49: 351-368.

41. Sonneville, P. (1976). Etude de la structure tridimensionelle des ecoulements autour d'un cylindre circulaire, Bulletin de la Direction des Etudes et Recherches, Serie A, No. 3 1976, Electricite de France.

42. Stern, F., Wilson, R., Shao, J. (2006), Quantitative V\&V of CFD simulations and certification of CFD codes. International journal for numerical methods in fluids 50:13351355.

43. Suh, J., Yang, J., Stern, F. (2011). The effect of air-water interface on the vortex shedding from a vertical circular cylinder. Journal of Fluids and Structures, 27: 1-22.

44. Sumer, B Mutlu. (2006). Hydrodynamics around cylindrical structures. World Scientific. 
45. Szepessy, S., P. W. Bearman (1992). Aspect ratio and end plate effects on vortex shedding from a circular cylinder. Journal of Fluid Mechanics.

46. Szepessy, S. (1994). On the spanwise correlation of vortex shedding from a circular cylinder at high subcritical Reynolds number. Physics of Fluids. 6: 2406-2416

47. Travin, A., M. Shur, M. Strelets and P. Spalart (2000). Detached-eddy simulations past a circular cylinder. Flow, Turbulence and Combustion, Springer. 63: 293-313.

48. Tropea, C. (1977), Boundary-layer Behavior on a Circular Cylinder for Critical Reynolds Numbers, PhD thesis, University of Toronto.

49. Vaz, G., C. Mabilat, R. van der Wal, P. Gallagher (2007). Viscous Flow Computations on a Smooth Cylinders: A Detailed Numerical Study With Validation. Proceedings of the 26th International Conference on Offshore Mechanics and Arctic Engineering.

50. Wang, Z., Suh, J., Yang, J., Stern, F. (2012), Sharp Interface LES of Breaking Waves by an Interface Piercing Body in Orthogonal Curvilinear Coordinates. 50th AIAA Paper.

51. Warschauer, K.A., Leene, J.A. (1971). Experiments on mean and fluctuating pressures of circular cylinders at cross flow at very high Reynolds numbers. Proceedings of International Conference on Wind Effects on Buildings and Structures.

52. Wei, T., Smith, C. R. (1986). Secondary vortices in the wake of circular cylinders. J. Fluid Mech. 169:513.

53. West, G. S., C. J. Apelt (1993). Measurements of Fluctuating Pressures and Forces on a Circular Cylinder in the Reynolds Number Range $10^{4}$ to $2.5 \times 10^{5}$. Journal of Fluids and Structures. 7: 227-244.

54. Wieselsberger, C. v. (1921). Neuere feststellungen über die gesetze des flüssigkeits-und luftwiderstands. Phys. Z. 22: 321-328.

55. Williamson, C. (1996). Vortex dynamics in the cylinder wake. Annual Review of Fluid Mechanics, Annual Reviews 4139 El Camino Way, PO Box 10139, Palo Alto, CA 943030139, USA. 28: 477-539.

56. Xing, T., Stern, F. (2010), Factors of safety for Richardson extrapolation. Journal of Fluids Engineering 132:061403.

57. Yang, J., E. Balaras (2006). An embedded-boundary formulation for large-eddy simulation of turbulent flows interacting with moving boundaries. Journal of Computational Physics, 215: $12-40$.

58. Yang, J., Stern. F (2009), Sharp interface immersed-boundary/level-set method for wavebody interactions. Journal of Computational Physics, Elsevier. 228: 6590--6616 
59. Ye, H., Zhong, Q., Wan, D. (2013). Benchmark Computations of Flows around a Stationary Cylinder with High Reynolds number by RANS-Overset Grid Approach. Proceedings of the 27th ITTC Workshop on Wave Run-up and Vortex Shedding.

60. Yeon, S., Yang, J., Stern, F. (2013). Large Eddy Simulation of Drag Crisis in Turbulent Flow Past a Circular Cylinder. Proceedings of the 27th ITTC Workshop on Wave Run-up and Vortex Shedding.

61. Yeon, S. (2014), Large-eddy simulation of sub-, critical and super-critical Reynolds number flow past a circular cylinder, PhD thesis, The University of Iowa. 
Table 1. Summary of experimental studies and validation variables

\begin{tabular}{|c|c|c|c|c|c|c|}
\hline EFD & $\begin{array}{c}\mathrm{Re} \\
\left(\mathrm{x} 10^{-5}\right)\end{array}$ & $\begin{array}{l}\text { Aspect ratio } \\
\text { (L/D) }\end{array}$ & $\begin{array}{l}\mathrm{Tu} \\
(\%)\end{array}$ & $\begin{array}{c}\text { Roughness } \\
\text { (k/D) }\end{array}$ & $\begin{array}{c}\text { Blockage } \\
(\%)\end{array}$ & Variables \\
\hline Wieselsberger (1921) & Sub-post & N/A & N/A & N/A & N/A & $\mathrm{C}_{\mathrm{D}}$ \\
\hline Achenbach (1968) & $0.6-50$ & Abt 3.3 & 0.7 & Smooth & 17 & $\mathrm{C}_{\mathrm{D}}, \theta_{s}, \mathrm{Cp}$ and $\mathrm{Cf}$ \\
\hline Schewe (1983) & $0.2-71$ & 10 & 0.4 & Smooth & 10 & $\mathrm{C}_{\mathrm{D}}, \mathrm{C}_{\mathrm{L}}, \mathrm{C}_{\mathrm{L}}^{\mathrm{rms}}$ and $\mathrm{St}$ \\
\hline MARIN exp. (Vaz et al., 2007) & Abt.0.3-8 & Abt 18.6 & N/A & N/A & N/A & $\mathrm{C}_{\mathrm{D}}$ \\
\hline Bearman (1969) & $1-7.5$ & 12 & 0.2 & Smooth & 6.5 & $\mathrm{C}_{\mathrm{D}}, \mathrm{C}_{\mathrm{L}},-\mathrm{Cpb}$ and $\mathrm{St}$ \\
\hline Norberg (1987) & $0.0005-2$ & 2 & 0.1 & Smooth & 1 & $-\mathrm{Cpb}$ \\
\hline Shih et al. (1993) & $3-80$ & Abt. 8.2 & N/A & $3.0 \times 10^{-4}$ & 11 & $-\mathrm{Cpb}$ \\
\hline Szepessy and Bearman (1992) & $0.08-1.4$ & 6.7 & 0.05 & N/A & 7.7 & $\mathrm{C}_{\mathrm{L}}^{\mathrm{rms}}$ \\
\hline West and Apelt (1993) & $0.11-2.2$ & $15-35$ & 0.2 & Smooth & 8.2 & $\mathrm{St}$ \\
\hline Roshko (1953) & $0.8-4.5$ & N/A & N/A & N/A & N/A & St \\
\hline Cantwell and Coles (1983) & $0.69-3.37$ & $>10$ & $<1$ & Smooth & $<10$ & $\mathrm{Cp}$ and $\mathrm{Cf}$ \\
\hline Bearman and Wadcock (1973) & 0.025 & N/A & N/A & N/A & N/A & $\Lambda$ \\
\hline Bruun and Davies (1975) & $0.6-6$ & 10 & 0.1 & Smooth & 13 & $\mathrm{R}_{\mathrm{pp}}, \Lambda$ \\
\hline Iida et al. (1997) & $0.06-1.5$ & N/A & N/A & N/A & N/A & $\Lambda$ \\
\hline Kacker et al. (1974) & $0.1-3$ & 8.0 & 0.4 & Smooth & 4.7 & $\Lambda$ \\
\hline Leehey and Hanson (1971) & $0.04-0.07$ & 97 & 0.04 & Smooth & Open jet & $\mathrm{R}_{\mathrm{pp}}, \Lambda$ \\
\hline Moeller (1982) & $0.05-0.56$ & $16 / 19$ & 0.3 & Smooth & Open jet & $\Lambda$ \\
\hline Novak and Tanaka (1977) & 0.02 & N/A & N/A & N/A & N/A & $\Lambda$ \\
\hline Sonneville (1976) & 0.45 & 13 & 0.4 & N/A & 5.6 & $\Lambda$ \\
\hline Szepessy (1994) & 0.43 & 10 & 0.05 & Smooth & 7.7 & $\Lambda$ \\
\hline Maekawa and Mizuno (1967) & $0.37-2.8$ & 3 & N/A & Smooth & 23 & $\mathrm{f}_{\mathrm{SL}}$ \\
\hline Bloor (1964) & $0.002-0.5$ & $20-655$ & 0.03 & Smooth & 5 & $f_{S L}$ \\
\hline Kourta et al. (1987) & $0.02-0.6$ & 7 & 0.1 & Smooth & 3 & $\mathrm{f}_{\mathrm{SL}}$ \\
\hline Okamoto et al. (1981) & $0.025-0.045$ & 4 & N/A & Smooth & 8 & $\mathrm{f}_{\mathrm{SL}}$ \\
\hline Wei and Smith (1986) & $0.012-0.11$ & $14-34$ & N/A & Smooth & $1-19$ & $\mathrm{f}_{\mathrm{SL}}$ \\
\hline
\end{tabular}


Table 2. Simualtion Reynolds numbers, grids, aspect ratio, convection scheme and wall distance

\begin{tabular}{|c|c|c|c|c|c|c|c|}
\hline \multicolumn{2}{|c|}{ Case } & $\operatorname{Re}$ & Grid & $N_{x} \times N_{r} \times N_{\theta}$ & $\begin{array}{c}\mathrm{AR} \\
\text { (L/D) }\end{array}$ & Method $\dagger$ & $y^{+}$ \\
\hline \multirow{13}{*}{$\begin{array}{l}\text { Sub- } \\
\text { critical }\end{array}$} & 1 & \multirow{7}{*}{$6.31 \times 10^{4}$} & Medium & $256 \times 512 \times 512=67 M$ & 2 & QUICK & \multirow{7}{*}{0.03} \\
\hline & 2 & & Medium & $256 \times 512 \times 512=67 M$ & 2 & WENO & \\
\hline & 3 & & Medium & $256 \times 512 \times 512=67 M$ & 4 & WENO & \\
\hline & 4 & & Fine & $512 \times 512 \times 512=134 M$ & 4 & WENO & \\
\hline & 5 & & Medium & $256 \times 512 \times 512=67 M$ & 8 & WENO & \\
\hline & 6 & & Coarse & $128 \times 256 \times 256=8 M$ & 8 & WENO & \\
\hline & 7 & & Medium & $256 \times 512 \times 512=67 M$ & 2 & NC-WENO & \\
\hline & 8 & \multirow{6}{*}{$1.26 \times 10^{5}$} & Medium & $256 \times 512 \times 512=67 M$ & 2 & QUICK & \multirow{6}{*}{0.05} \\
\hline & 9 & & Medium & $256 \times 512 \times 512=67 M$ & 2 & WENO & \\
\hline & 10 & & Medium & $256 \times 512 \times 512=67 M$ & 4 & WENO & \\
\hline & 11 & & Fine & $512 \times 512 \times 512=134 M$ & 4 & WENO & \\
\hline & 12 & & Medium & $256 \times 512 \times 512=67 M$ & 8 & WENO & \\
\hline & 13 & & Medium & $256 \times 512 \times 512=67 M$ & 2 & NC-WENO & \\
\hline \multirow{6}{*}{ Critical } & 14 & \multirow{6}{*}{$2.52 \times 10^{5}$} & Medium & $256 \times 512 \times 512=67 M$ & 2 & QUICK & \multirow{6}{*}{0.08} \\
\hline & 15 & & Medium & $256 \times 512 \times 512=67 M$ & 2 & WENO & \\
\hline & 16 & & Medium & $256 \times 512 \times 512=67 M$ & 4 & WENO & \\
\hline & 17 & & Fine & $512 \times 512 \times 512=134 M$ & 4 & WENO & \\
\hline & 18 & & Medium & $256 \times 512 \times 512=67 M$ & 8 & WENO & \\
\hline & 19 & & Medium & $256 \times 512 \times 512=67 M$ & 2 & NC-WENO & \\
\hline \multirow{11}{*}{$\begin{array}{l}\text { Super- } \\
\text { critical }\end{array}$} & 20 & $4.15 \times 10^{5}$ & Medium & $256 \times 512 \times 512=67 M$ & 2 & QUICK & 0.10 \\
\hline & 21 & \multirow{2}{*}{$5.06 \times 10^{5}$} & Medium & $256 \times 512 \times 512=67 M$ & 2 & QUICK & \multirow{2}{*}{0.14} \\
\hline & 22 & & Medium & $256 \times 512 \times 512=67 M$ & 2 & NC-WENO & \\
\hline & 23 & \multirow{8}{*}{$7.57 \times 10^{5}$} & $\begin{array}{l}\text { Medium } \\
\text { (F) }\end{array}$ & $256 \times 512 \times 512=67 M$ & 2 & QUICK & 0.15 \\
\hline & 24 & & $\mathrm{M}$ & $128 \times 256 \times 256=8 \mathrm{M}$ & 2 & QUICK & 0.33 \\
\hline & 25 & & $\mathrm{C}$ & $64 \times 128 \times 128=1 M$ & 2 & QUICK & 0.67 \\
\hline & 26 & & Medium & $256 \times 512 \times 512=67 M$ & 2 & WENO & \multirow{5}{*}{0.15} \\
\hline & 27 & & Medium & $256 \times 512 \times 512=67 M$ & 4 & WENO & \\
\hline & 28 & & Fine & $512 \times 512 \times 512=134 M$ & 4 & WENO & \\
\hline & 29 & & Medium & $256 \times 512 \times 512=67 M$ & 8 & WENO & \\
\hline & 30 & & Medium & $256 \times 512 \times 512=67 M$ & 2 & NC-WENO & \\
\hline
\end{tabular}

$\dagger$ NC-WENO is Non-conservative WENO scheme. QUICK and WENO are conservative schemes.

$\dagger \dagger$ Color coded cases are to be submitted to ITTC. Detailed analysis is carried out with purple color. 
Table 3. Summary of integral and local variables with experimental data

\begin{tabular}{|c|c|c|c|c|c|c|c|c|c|}
\hline & $\operatorname{Re}$ & & $C_{D}$ & $\theta_{\mathrm{LS}}$ & $\theta_{\mathrm{TS}}$ & $-\mathrm{C}_{\mathrm{pb}}$ & $\mathrm{C}_{\mathrm{L}}^{\mathrm{RMS}}$ & St & $\Lambda / \mathrm{D}$ \\
\hline \multirow{4}{*}{ Sub-critical } & $6.31 \times 10^{4}$ & D & 1.20 & 81 & - & 1.30 & 0.55 & 0.20 & 3.5 \\
\hline & & $\mathrm{S}$ & 1.37 & 82 & - & 1.46 & 0.60 & 0.19 & 3.3 \\
\hline & $1.26 \times 10^{5}$ & D & 1.16 & 75 & - & 1.32 & 0.56 & 0.20 & 3.0 \\
\hline & & $\mathrm{S}$ & 1.37 & 81 & - & 1.64 & 0.62 & 0.20 & 2.9 \\
\hline \multirow{2}{*}{ Critical } & $2.52 \times 10^{5}$ & $\mathrm{D}$ & 0.92 & 94 & - & 1.00 & 0.17 & 0.19 & 2.4 \\
\hline & & $\mathrm{S}$ & 0.56 & 89 & - & 0.44 & 0.12 & 0.19 & 0.8 \\
\hline \multirow{6}{*}{$\begin{array}{l}\text { Super- } \\
\text { critical }\end{array}$} & $4.15 \times 10^{5}$ & $\mathrm{D}$ & 0.36 & - & 136 & 0.24 & 0.021 & 0.47 & 1.2 \\
\hline & & $\mathrm{S}$ & 0.27 & 95 & 113 & 0.30 & 0.064 & 0.41 & - \\
\hline & $5.06 \times 10^{5}$ & D & 0.29 & - & 139 & 0.24 & 0.023 & 0.47 & 1.1 \\
\hline & & S & 0.25 & 96 & 113 & 0.25 & 0.061 & 0.41 & - \\
\hline & $7.57 \times 10^{5}$ & D & 0.23 & - & 135 & 0.24 & 0.027 & 0.46 & 1.1 \\
\hline & & $\mathrm{S}$ & 0.21 & 94 & 112 & 0.34 & 0.042 & 0.34 & 1.3 \\
\hline
\end{tabular}

Table 4. Errors of integral and local variables

\begin{tabular}{|c|c|c|c|c|c|c|c|c|c|}
\hline & \multirow{2}{*}{$\operatorname{Re}$} & \multicolumn{2}{|c|}{$\mathrm{C}_{\mathrm{D}}$} & \multirow{2}{*}{$\begin{array}{c}\theta_{\mathrm{LS}} \\
\mathrm{E} \% \mathrm{~T}\end{array}$} & \multirow{2}{*}{$\begin{array}{c}\theta_{\mathrm{TS}} \\
\mathrm{E} \% \mathrm{~T}\end{array}$} & \multirow{2}{*}{$\begin{array}{l}-\mathrm{C}_{\mathrm{pb}} \\
\mathrm{E} \% \mathrm{~T}\end{array}$} & \multirow{2}{*}{$\begin{array}{l}\mathrm{C}_{\mathrm{L}}{ }^{\mathrm{RMS} S} \\
\mathrm{E} \% \mathrm{~T}\end{array}$} & \multirow{2}{*}{$\begin{array}{c}\text { St } \\
\mathrm{E} \% \mathrm{~T}\end{array}$} & \multirow{2}{*}{$\begin{array}{l}\Lambda / \mathrm{D} \\
\mathrm{E} \% \mathrm{~T}\end{array}$} \\
\hline & & $\mathrm{E} \% \mathrm{~T}$ & $\mathrm{U}_{\mathrm{FB}} \% \mathrm{~T}$ & & & & & & \\
\hline \multirow{3}{*}{ Sub-critical } & $6.31 \times 10^{4}$ & 14 & 9 & 2 & - & 12 & 8 & -5 & -6 \\
\hline & $1.26 \times 10^{5}$ & 18 & 7 & 9 & - & 24 & 10 & 0 & -3 \\
\hline & Average & 16 & 8 & 5 & & 18 & 9 & 3 & 5 \\
\hline Critical & $2.52 \times 10^{5}$ & -39 & 9 & -8 & - & -56 & -8 & 0 & -67 \\
\hline \multirow{4}{*}{$\begin{array}{l}\text { Super- } \\
\text { critical }\end{array}$} & $4.15 \times 10^{5}$ & -25 & 21 & - & -36 & 25 & 7 & -13 & - \\
\hline & $5.06 \times 10^{5}$ & -14 & 6 & - & -41 & 4 & 6 & -13 & - \\
\hline & $7.57 \times 10^{5}$ & -4 & 5 & - & -36 & 42 & 3 & -26 & 18 \\
\hline & Average & 14 & 11 & - & 38 & 24 & 5 & 17 & 18 \\
\hline \multicolumn{2}{|c|}{ Total average } & 23 & 10 & 6 & 38 & 33 & 7 & 7 & 30 \\
\hline
\end{tabular}

Table 5. Key point angles from Achenbach (1968) and Pfeil \& Orth (1990) (super-critical)

\begin{tabular}{ccccccc}
\hline & $\operatorname{Re}$ & $\begin{array}{c}\theta_{\mathrm{MP}} \\
(\mathrm{deg})\end{array}$ & $\begin{array}{c}\theta_{\mathrm{LS}} \\
(\mathrm{deg})\end{array}$ & $\begin{array}{c}\theta_{\mathrm{TT}} \\
(\mathrm{deg})\end{array}$ & $\begin{array}{c}\theta_{\mathrm{TR}} \\
(\mathrm{deg})\end{array}$ & $\begin{array}{c}\theta_{\mathrm{TS}} \\
(\mathrm{deg})\end{array}$ \\
\hline Sub-critical & $1.0 \times 10^{5}$ & 75 & 80 & & & \\
\hline Critical & $2.1 \times 10^{5}$ & 78 & 89 & & & \\
& $2.6 \times 10^{5}$ & 74 & 94 & & & 120 \\
\hline Super-critical & $8.5 \times 10^{5}$ & 110 & 110 & & 148 \\
\hline Super-critical & $6.0 \times 10^{5}$ & & 100 & 110 & 114 & \\
\hline Post-critical & $3.6 \times 10^{6}$ & 79 & & & & 110 \\
\hline
\end{tabular}


Table 6. Key points of the present LES study

\begin{tabular}{ccccccc}
\hline & $\operatorname{Re}$ & $\begin{array}{c}\theta_{\mathrm{MP}} \\
(\mathrm{deg})\end{array}$ & $\begin{array}{c}\theta_{\mathrm{LS}} \\
(\mathrm{deg})\end{array}$ & $\begin{array}{c}\theta_{\mathrm{TT}} \\
(\mathrm{deg})\end{array}$ & $\begin{array}{c}\theta_{\mathrm{TR}} \\
(\mathrm{deg})\end{array}$ & $\begin{array}{c}\theta_{\mathrm{TS}} \\
(\mathrm{deg})\end{array}$ \\
\hline Sub-critical & $6.31 \times 10^{4}$ & 69 & 82 & & & \\
& $1.26 \times 10^{5}$ & 69 & 81 & & & \\
Critical & $2.52 \times 10^{5}$ & 77 & 89 & & & \\
\hline \multirow{3}{*}{ Super-critical } & $4.15 \times 10^{5}$ & 82 & 95 & 102 & 104 & 111 \\
& $5.06 \times 10^{6}$ & 83 & 96 & 102 & 104 & 111 \\
& $7.57 \times 10^{6}$ & 82 & 94 & 102 & 105 & 110 \\
\hline
\end{tabular}

Table 7. Errors for key points of the present LES study

\begin{tabular}{|c|c|c|c|c|c|}
\hline & $\operatorname{Re}$ & $\begin{array}{r}\theta_{\mathrm{MP}} \\
\mathrm{E} \% \mathrm{~T}\end{array}$ & $\begin{array}{c}\theta_{\mathrm{LS}} \\
\mathrm{E} \% \mathrm{~T}\end{array}$ & $\begin{array}{c}\theta_{\mathrm{TR}} \\
\mathrm{E} \% \mathrm{~T}\end{array}$ & $\begin{array}{c}\theta_{\mathrm{TS}} \\
\mathrm{E} \% \mathrm{~T}\end{array}$ \\
\hline \multirow{3}{*}{ Sub-critical } & $6.31 \times 10^{4}$ & -8 & 2 & & \\
\hline & $1.26 \times 10^{5}$ & -12 & 9 & & \\
\hline & Average & 10 & 5 & & \\
\hline Critical & $2.52 \times 10^{5}$ & 4 & -8 & & \\
\hline \multirow{4}{*}{ Super-critical } & $4.15 \times 10^{5}$ & -25 & - & -15 & -36 \\
\hline & $5.06 \times 10^{6}$ & -25 & - & -15 & -41 \\
\hline & $7.57 \times 10^{6}$ & -25 & - & -15 & -36 \\
\hline & Average & 25 & & 15 & 38 \\
\hline \multicolumn{2}{|c|}{ Total average } & 17 & 6 & 15 & 38 \\
\hline
\end{tabular}

Table 8. Comparison of key points and thickness of separation bubble

\begin{tabular}{ccccccc}
\hline & $\mathrm{Re}$ & $\begin{array}{c}\theta_{\mathrm{LS}} \\
(\mathrm{deg})\end{array}$ & $\begin{array}{c}\theta_{\mathrm{TT}} \\
(\mathrm{deg})\end{array}$ & $\begin{array}{c}\theta_{\mathrm{TR}} \\
(\mathrm{deg})\end{array}$ & $\begin{array}{c}\theta_{\mathrm{TS}} \\
(\mathrm{deg})\end{array}$ & $\begin{array}{c}\text { Thickness } \\
(\mathrm{mm})\end{array}$ \\
\hline $\begin{array}{c}\text { Experiment (Pfeil \& Orth, 1990) } \\
\text { Present LES }\end{array}$ & $6.00 \times 10^{5}$ & 100 & 110 & 114 & - & 0.05 \\
\hline E\%T & $7.57 \times 10^{5}$ & 94 & 102 & 105 & 110 & 0.05 \\
\hline & & -6 & -7 & -8 & - & - \\
\hline
\end{tabular}




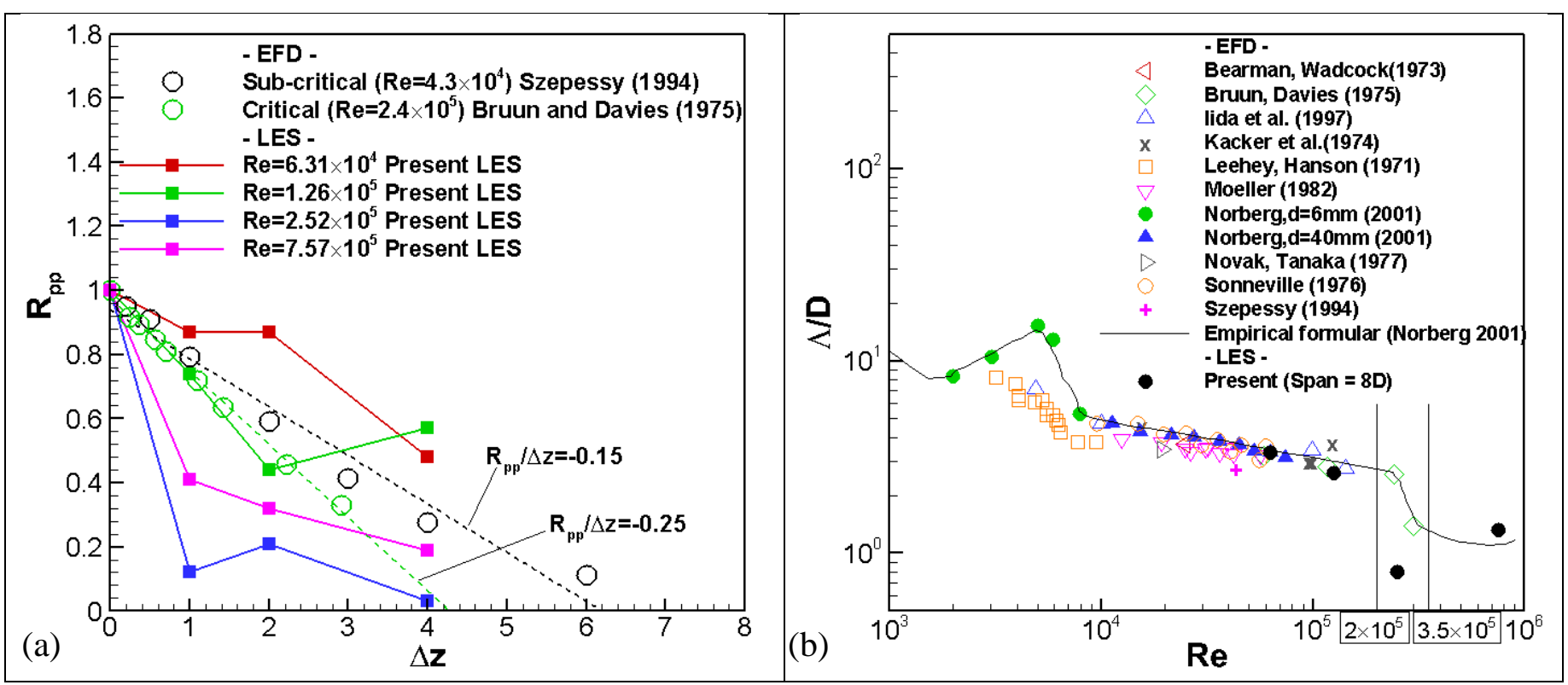

Figure 1. Comparison of correlation coefficient and length. (a) Spanwise cross correlation of pressure against separation from midspan; (b) Spanwise correlation length against Re. 

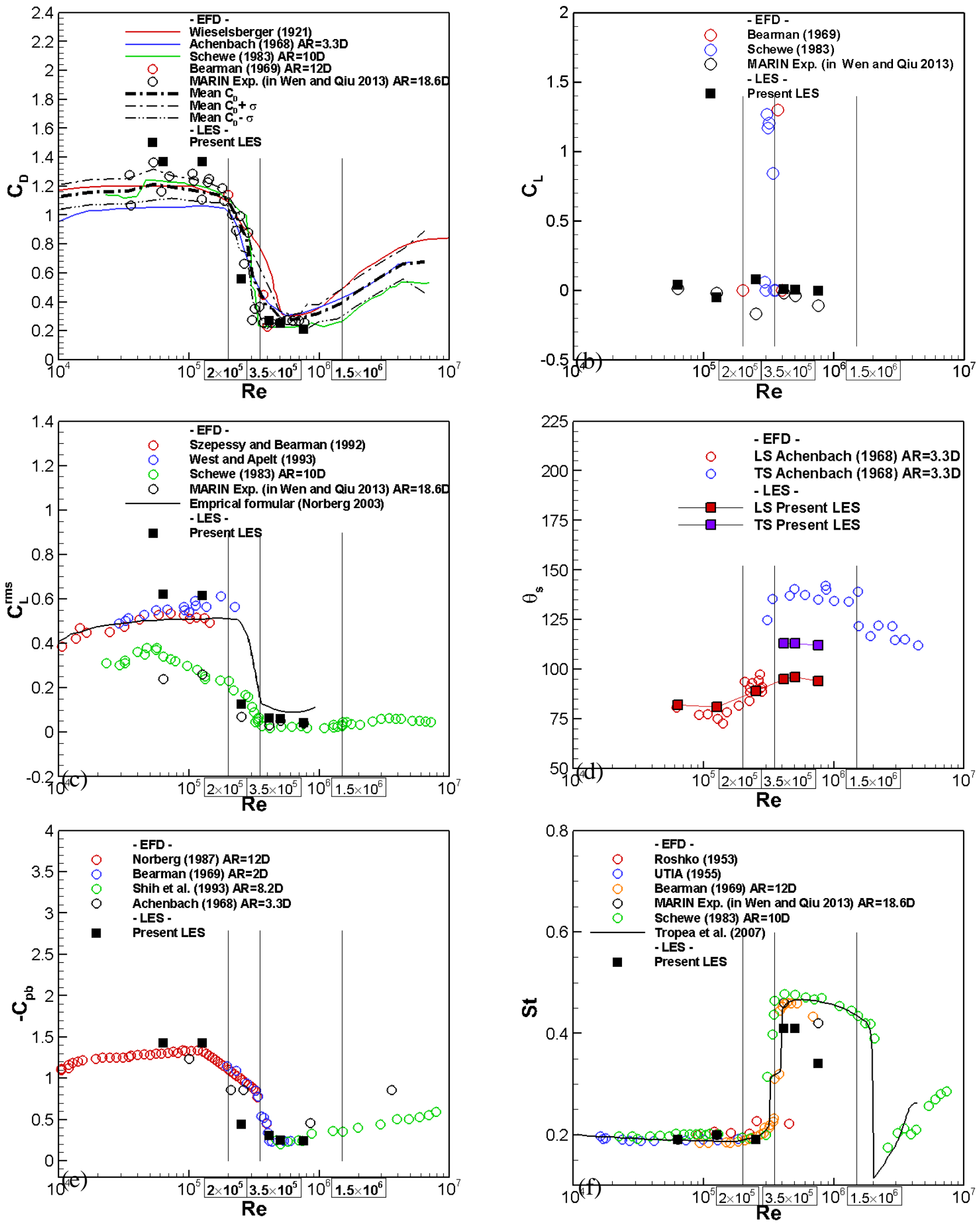

Figure 2. Drag (a), lift (b), RMS lift (c) coefficients, separation angle (d), base pressure (e) and Strouhal number (f) as functions of Re. 

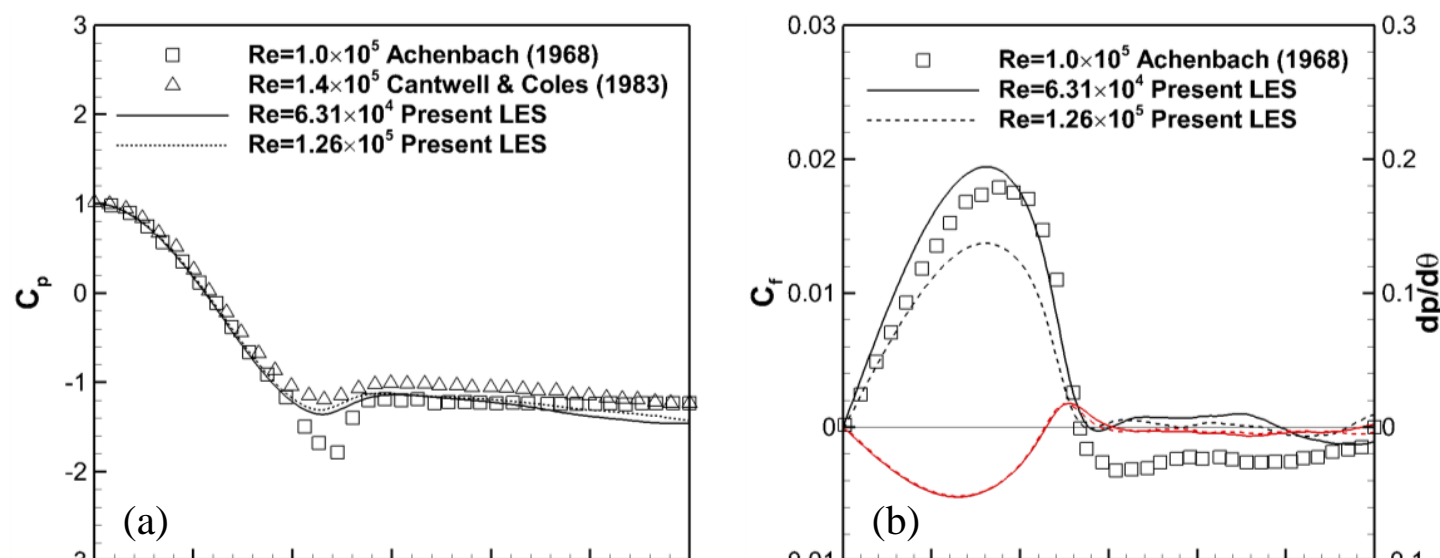

Sub-critical
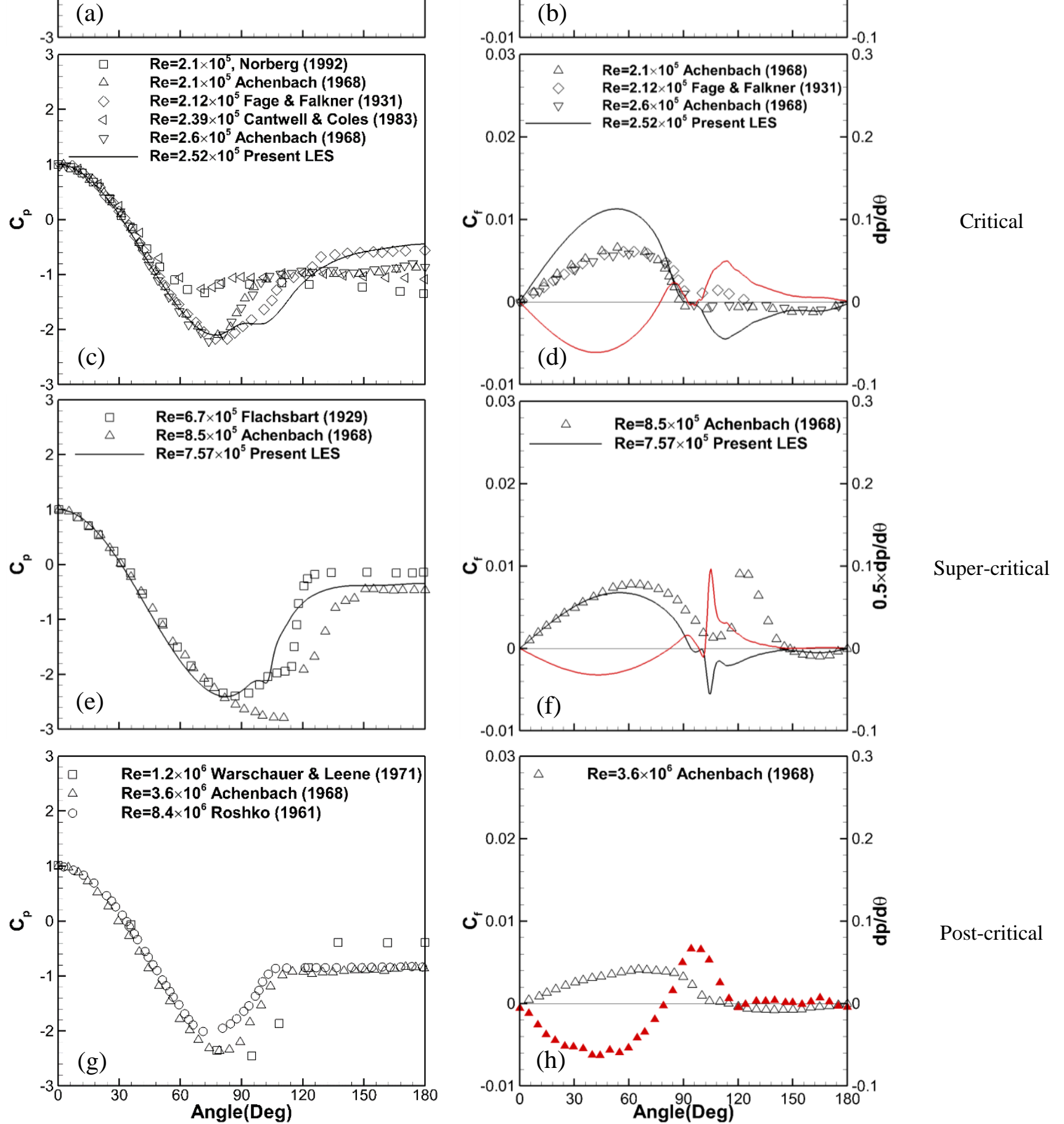

Figure 3. Pressure (left) and skin-friction with pressure gradient in red (right). 

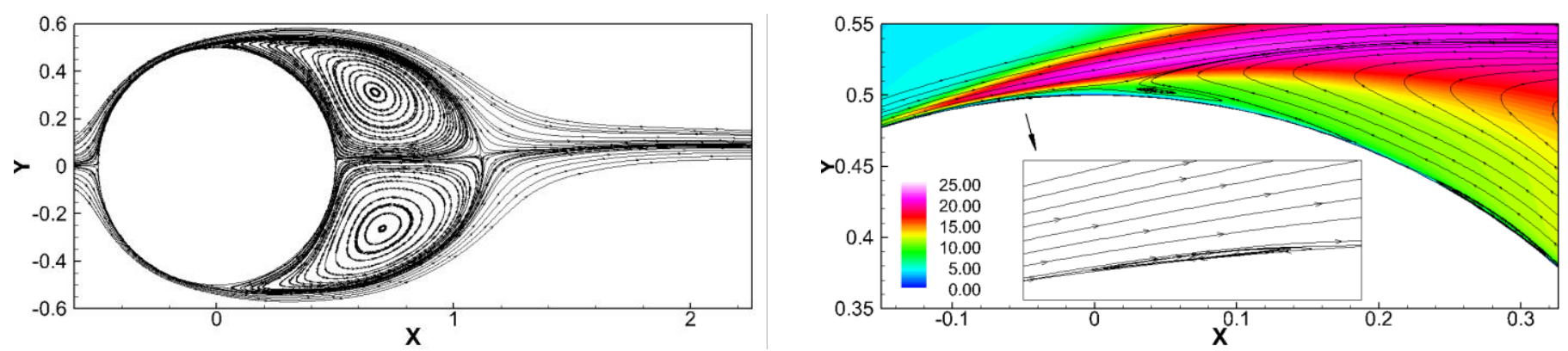

(a) $\operatorname{Re}=1.26 \times 10^{5}$
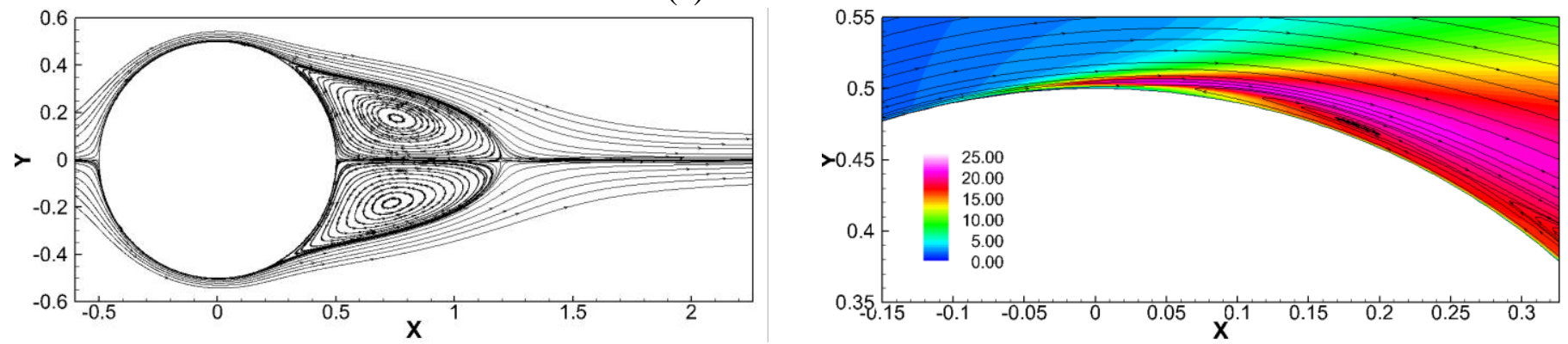

(b) $\mathrm{Re}=2.52 \times 10^{5}$
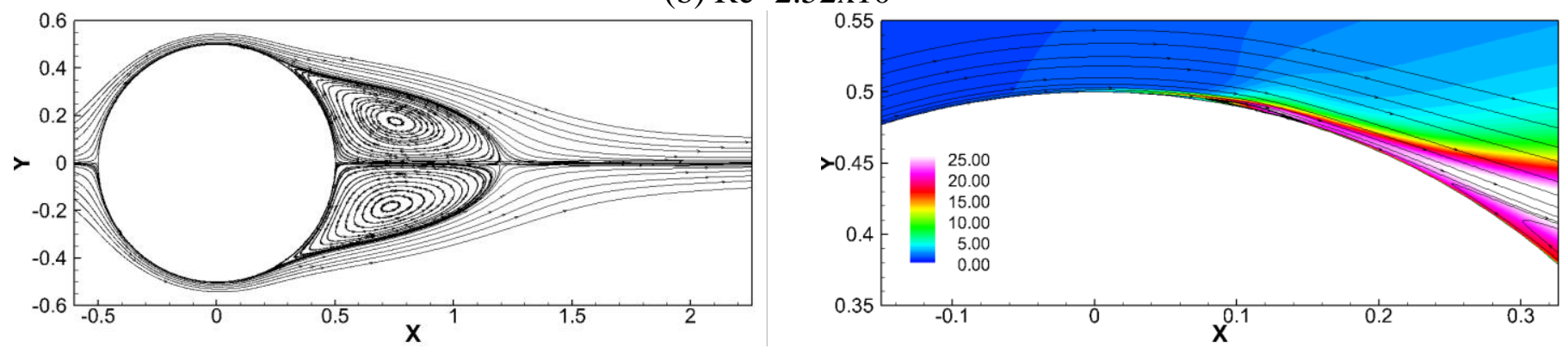

(c) $\operatorname{Re}=7.57 \times 10^{5}$

Figure 4. Mean streamlines with turbulent intensity contours (right). 


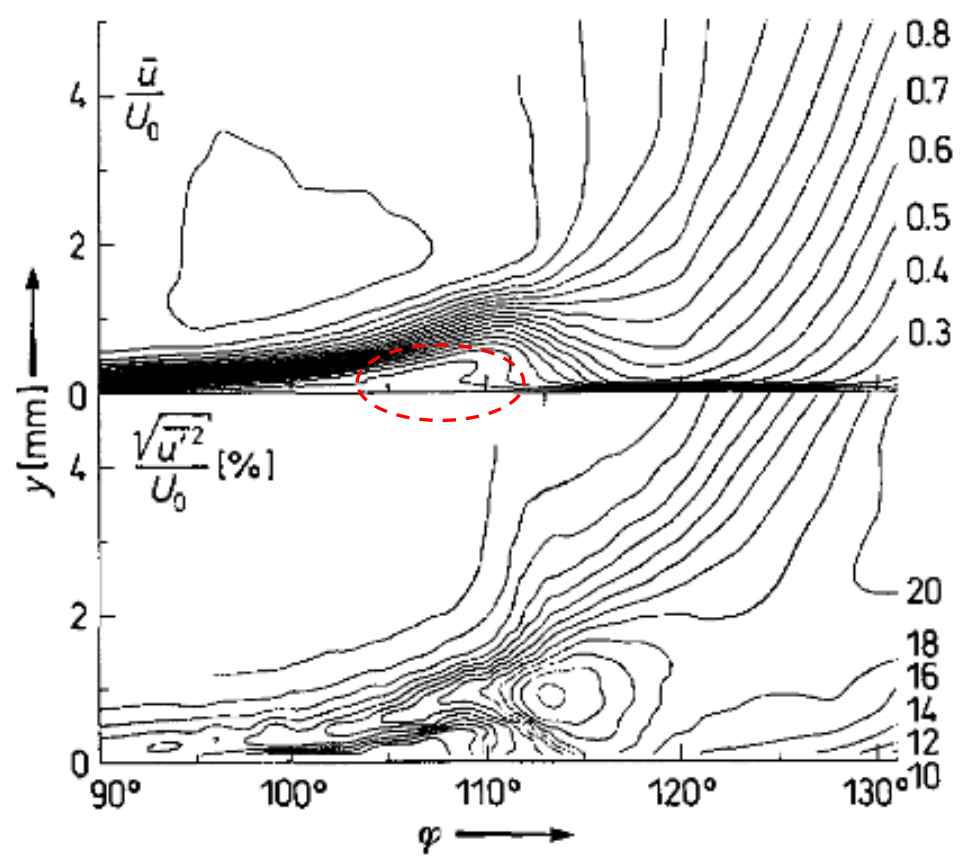

(a) $\operatorname{Re}=6 \times 10^{5}$ (Pfeil and Orth, 1990)
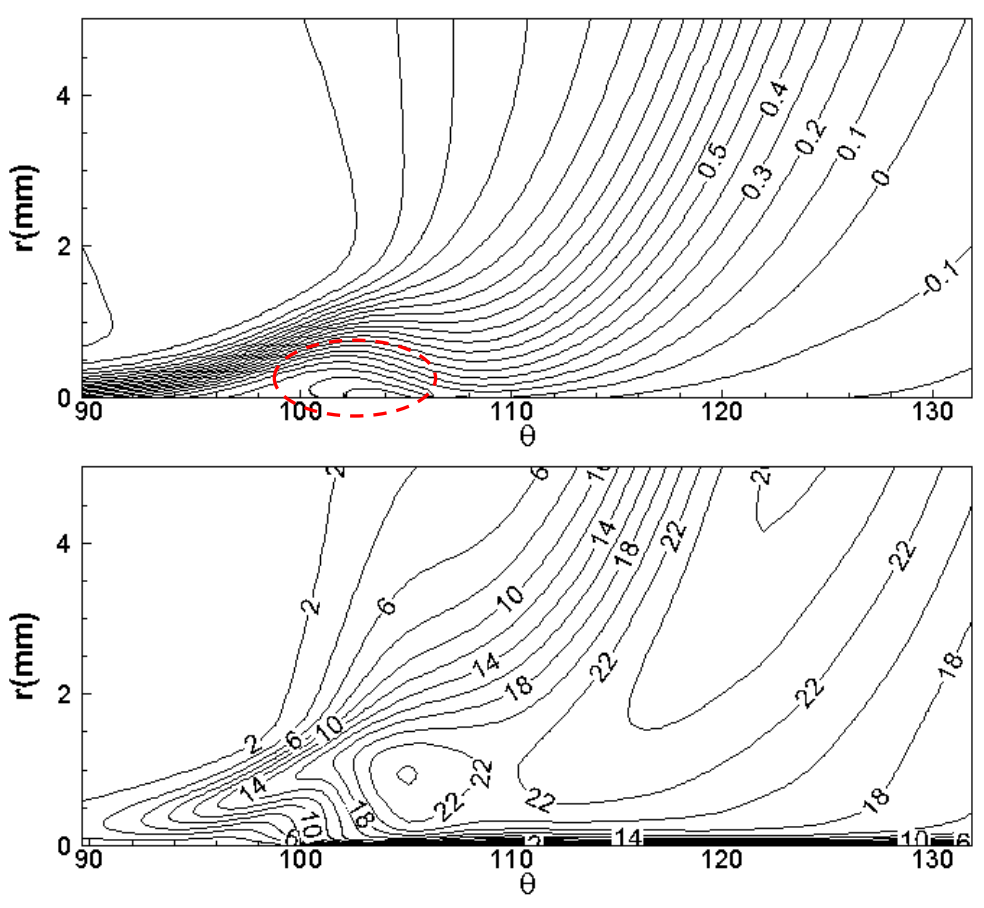

(b) $\mathrm{Re}=7.57 \times 10^{5} \mathrm{LES}$

Figure 5. Mean velocity (top) and turbulence intensity (bottom) for laminar separation bubble. The wallnormal distance is dimensionalized with $\mathrm{D}=300 \mathrm{~mm}$. 


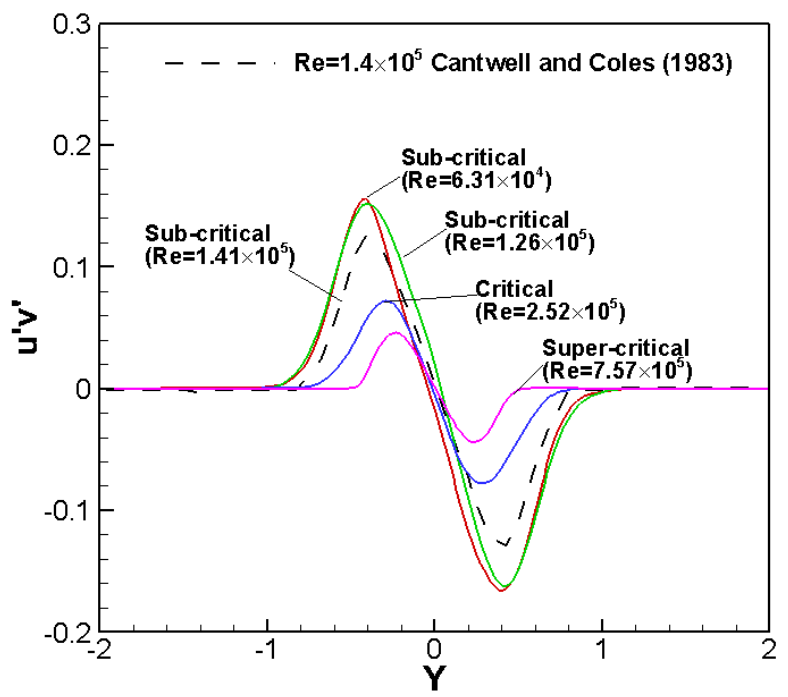

(a) Shear component along $\mathrm{x}=1$

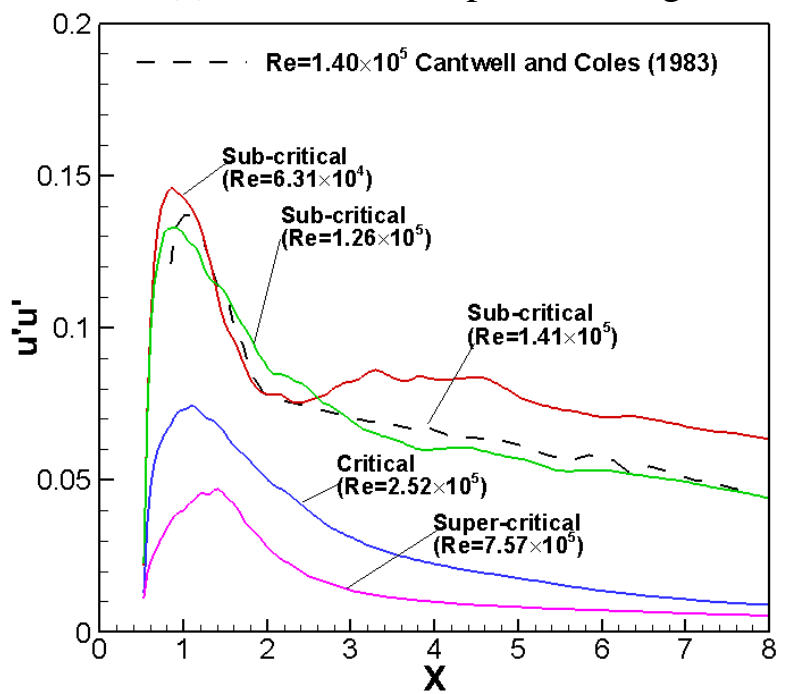

(b) Streamwise component along $\mathrm{y}=0$

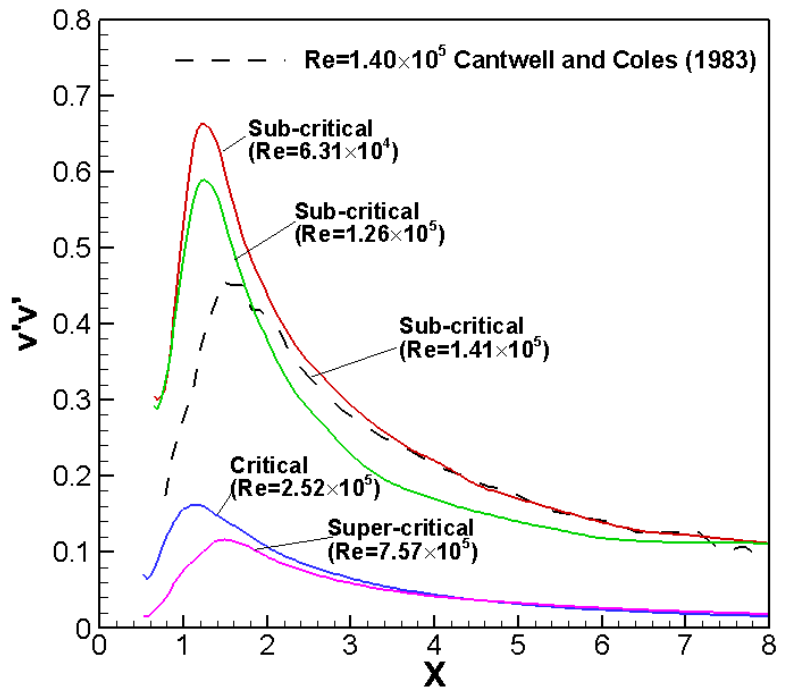

(c) Transverse component along $\mathrm{y}=0$

Figure 6. Variation of Reynolds stresses with Re. 

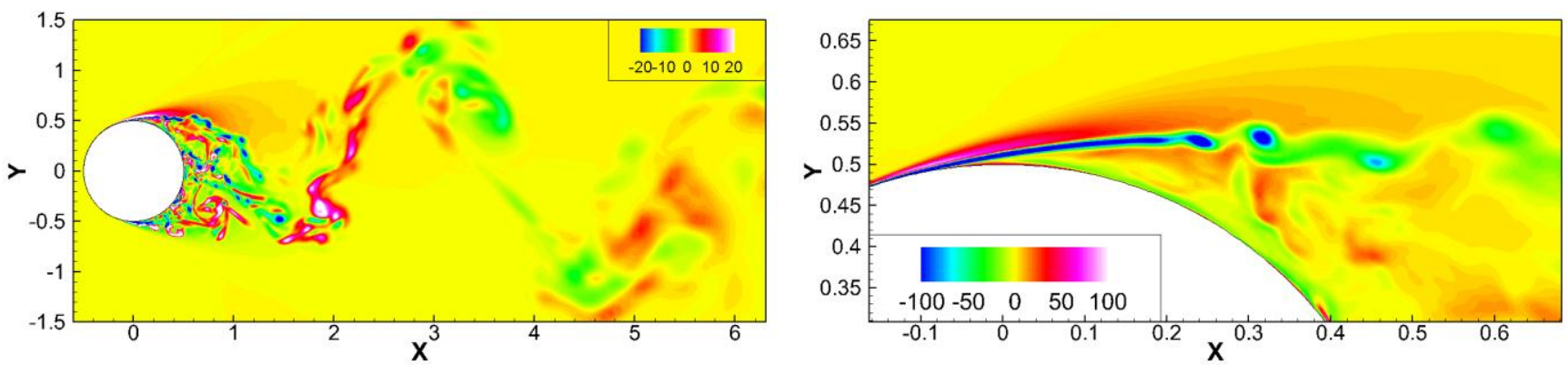

(a) $\operatorname{Re}=1.26 \times 10^{5}$
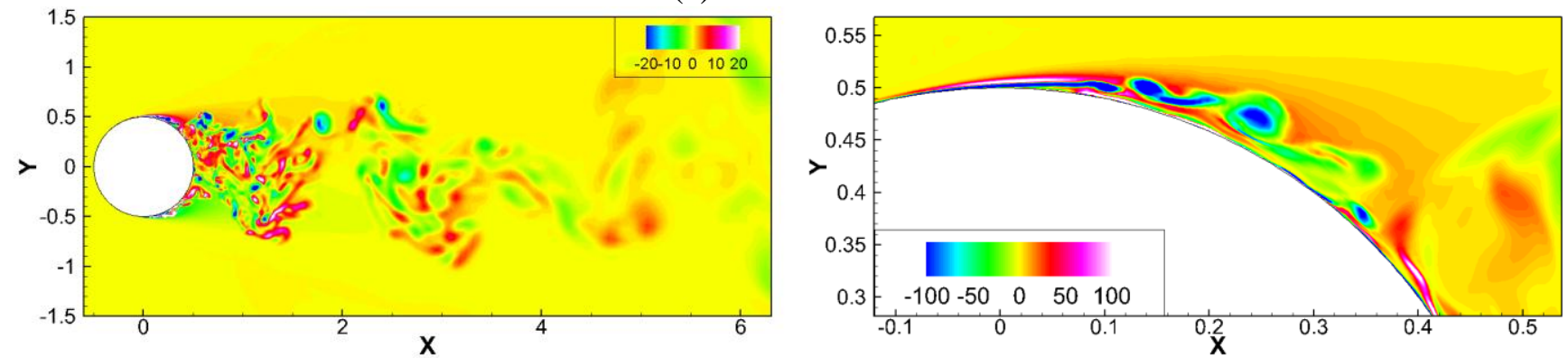

(b) $\operatorname{Re}=2.52 \times 10^{5}$
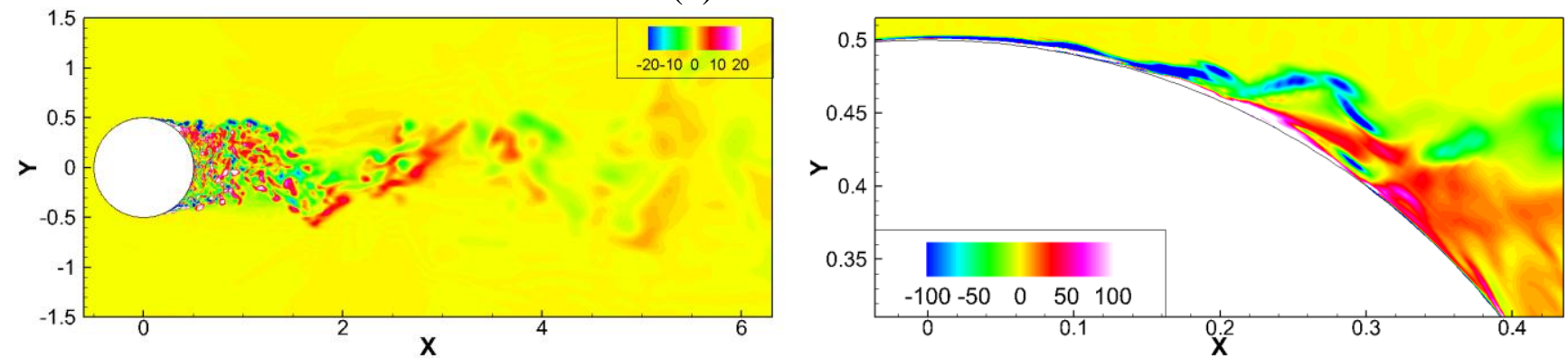

(c) $\operatorname{Re}=7.57 \times 10^{5}$

Figure 7. Instantaneous spanwise vorticity contours, right side shows the close-up views. 


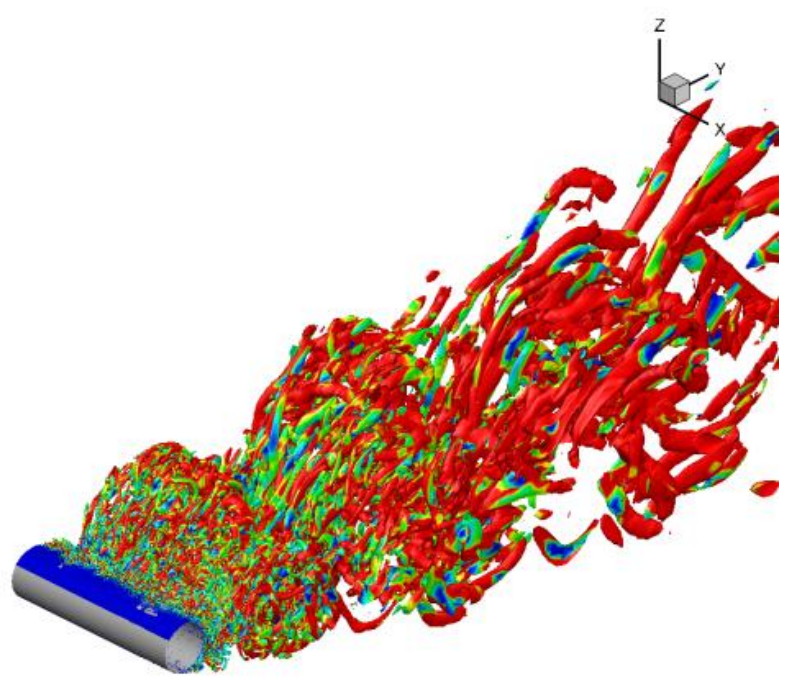

(a) sub-critical $\left(\operatorname{Re}=1.26 \times 10^{5}\right)$

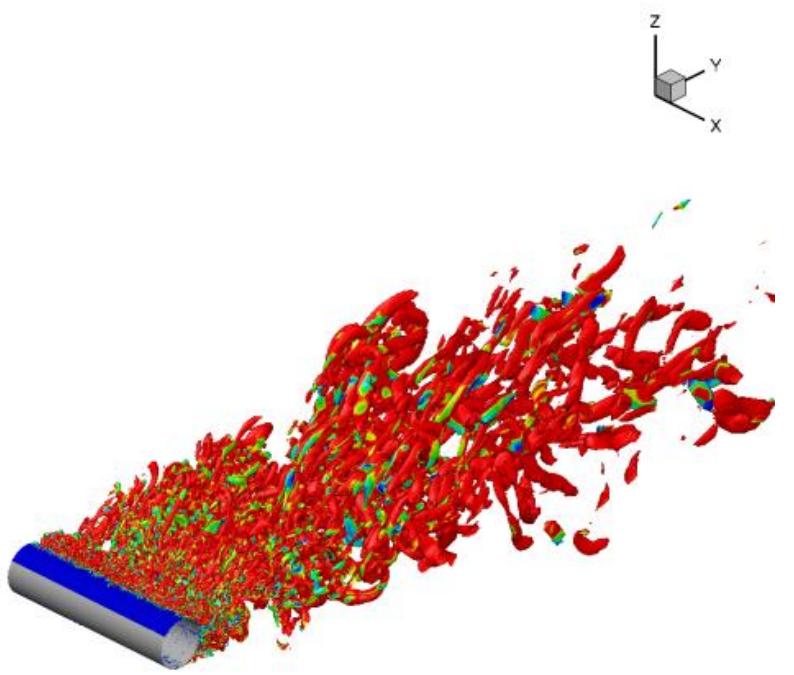

(b) critical $\left(\operatorname{Re}=2.52 \times 10^{5}\right)$
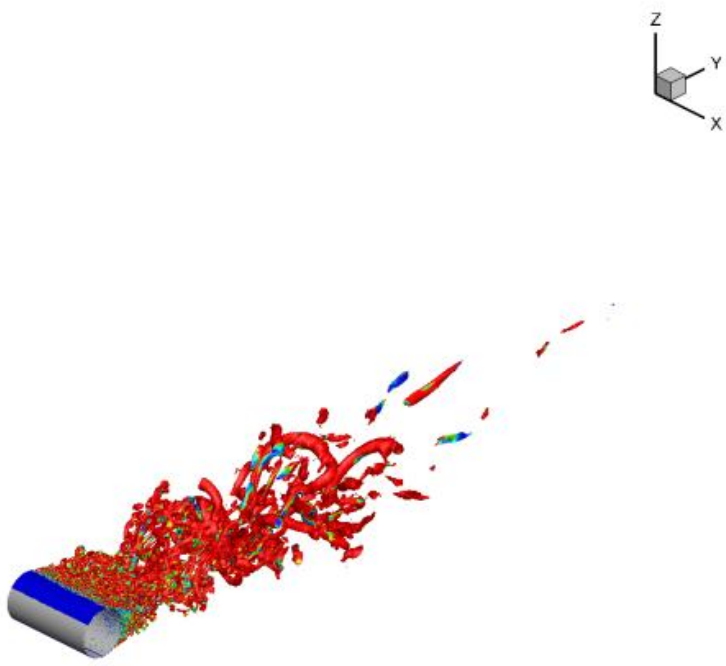

(c) super-critical $\left(\mathrm{Re}=7.57 \times 10^{5}\right)$

Figure 8. Vortex structures with isosurfaces of Q-criterion colored by $\boldsymbol{v}_{\boldsymbol{t}} / \boldsymbol{v}$. 